\title{
CHIANTI-AN ATOMIC DATABASE FOR EMISSION LINES. VII. NEW DATA FOR X-RAYS AND OTHER IMPROVEMENTS
}

\author{
E. LANDI \\ Artep, Inc., Ellicott City, MD 21042; and Naval Research Laboratory, 4555 Overlook Avenue SW, Washington, DC 20375-5320 \\ G. Del Zanna \\ Mullard Space Science Laboratory, Holmbury St. Mary, Dorking RH5 6NT, UK \\ P. R. Young \\ CCLRC Rutherford Appleton Laboratory, Chilton, Didcot OX11 0QX, UK \\ K. P. DERE \\ School of Computational Sciences, George Mason University, MS 5C3, 4400 University Drive, Fairfax, VA 22030 \\ H. E. MASON \\ Department of Applied Mathematics and Theoretical Physics, University of Cambridge, Wilberforce Road, Cambridge CB3 0WA, UK \\ AND \\ M. LANDINI \\ Dipartimento di Astronomia e Scienza dello Spazio, Universitá di Firenze, Largo E. Fermi 2, 50125 Florence, Italy \\ Received 2005 July 22; accepted 2005 September 19
}

\begin{abstract}
The CHIANTI atomic database contains atomic energy levels, wavelengths, radiative transition probabilities, and collisional excitation data for a large number of ions of astrophysical interest. CHIANTI also includes a suite of IDL routines to calculate synthetic spectra and carry out plasma diagnostics. Version 5 has been released, which includes several new features, as well as new data for many ions. The new features in CHIANTI are as follows: the inclusion of ionization and recombination rates to individual excited levels as a means to populate atomic levels; data for $\mathrm{K} \alpha$ and $\mathrm{K} \beta$ emission from $\mathrm{Fe}$ II to $\mathrm{Fe}$ xxIv; new data for high-energy configurations in $\mathrm{Fe}$ xVII to Fe xxIII; and a complete reassessment of level energies and line identifications in the X-ray range, multitemperature particle distributions, and photoexcitation from any user-defined radiation field. New data for ions already in the database, as well as data for ions not present in earlier versions of the database, are also included. Version 5 of CHIANTI represents a major improvement in the calculation of line emissivities and synthetic spectra in the X-ray range and expands and improves theoretical spectra calculations in all other wavelength ranges.
\end{abstract}

Subject headings: atomic data - plasmas

\section{INTRODUCTION}

This paper describes the new version (version 5) of the CHIANTI database. CHIANTI, first released in 1996 (Dere et al. 1997), consists of a database of atomic data and transition rates necessary to calculate line and continuum emissivities from optically thin plasmas. CHIANTI also includes a suite of computer programs that allow the computation of synthetic spectra and the application of plasma diagnostic techniques to observed spectra. All the data in the CHIANTI database are selected from the refereed literature after being critically assessed in order to ascertain their reliability and accuracy.

Since the first release, CHIANTI has enjoyed extraordinary success and has been used by a continuously growing number of scientists around the world in all the disciplines of astrophysics where spectroscopy is involved. CHIANTI has become the standard database for the solar physics community and has been referenced in more than 100 papers from solar physicists.

CHIANTI emissivities have been used in stellar physics as well, to study the coronae of cool stars (i.e., Dupree et al. 2005b; Pagano et al. 2004; Sim \& Jordan 2003; Jordan et al. 2001), flare stars (Maggio et al. 2004; Howley et al. 2003; Bloomfield et al. 2002; Robinson et al. 2001), and young stars (Matranga et al. 2005). CHIANTI has also been used to determine the physical parameters of T Tauri stars (Lamzin et al. 2001, 2004; Argiroffi et al. 2004; Drake \& Sarna 2003; Alexander et al. 2005; Hartigan et al. 2004), their winds (Dupree et al. 2005a), and circumstellar environment (Walter et al. 2003). Spectra from binary systems such as Algol (Ness et al. 2002; Schmitt \& Ness 2004; Drake 2003; Chung et al. 2004), Capella (Phillips et al. 2001), and 44 Bootis (Gondoin 2004) have been investigated with CHIANTI, along with data from M-type Mira stars (Richter et al. 2003), classical novae (Mauche \& Raymond 2000; Petz et al. 2005), H-R gap giants (Scelsi et al. 2004), white dwarfs (Werner et al. 2004a, 2004b; Drake \& Werner 2005), symbiotic stars (Young et al. 2005a, 2005b), M dwarfs (Fuhrmeister et al. 2004), wind from hot stars (Herald et al. 2005), and the Arches cluster of massive stars (Raga et al. 2001).

CHIANTI data have been applied also to studies of nonstellar objects, such as supernova remnants (Smith \& Cox 2001; Sankrit et al. 2003; Velazquez et al. 2003, 2004), interstellar medium (Arthur 2001; Edelstein et al. 2001; Hurwitz et al. 2005), HerbigHaro objects (Raga et al. 2002), the Io plasma torus (Herbert et al. 2001; Feldman et al. 2001, 2004), the shock-cloud interaction in the Cygnus Loop (Danforth et al. 2001), and galaxies (Dixon et al. 2001; Swartz et al. 2002; Matsumoto et al. 2004; Lee \& Hwang 2004).

The accuracy and completeness of the CHIANTI database has led other databases to include CHIANTI data, such as APEC/APED (Smith et al. 2001), XSTAR (Bautista \& Kallman 2001), PintOfAle 
TABLE 1

Ions InCLuded in the CHIANTI Database

\begin{tabular}{|c|c|c|c|c|c|c|c|c|c|c|c|c|c|c|c|c|}
\hline Ion & I & II & III & IV & $\mathrm{V}$ & VI & VII & VIII & IX & $\mathrm{X}$ & $\mathrm{XI}$ & XII & XIII & XIV & $\mathrm{XV}$ & XVI \\
\hline
\end{tabular}

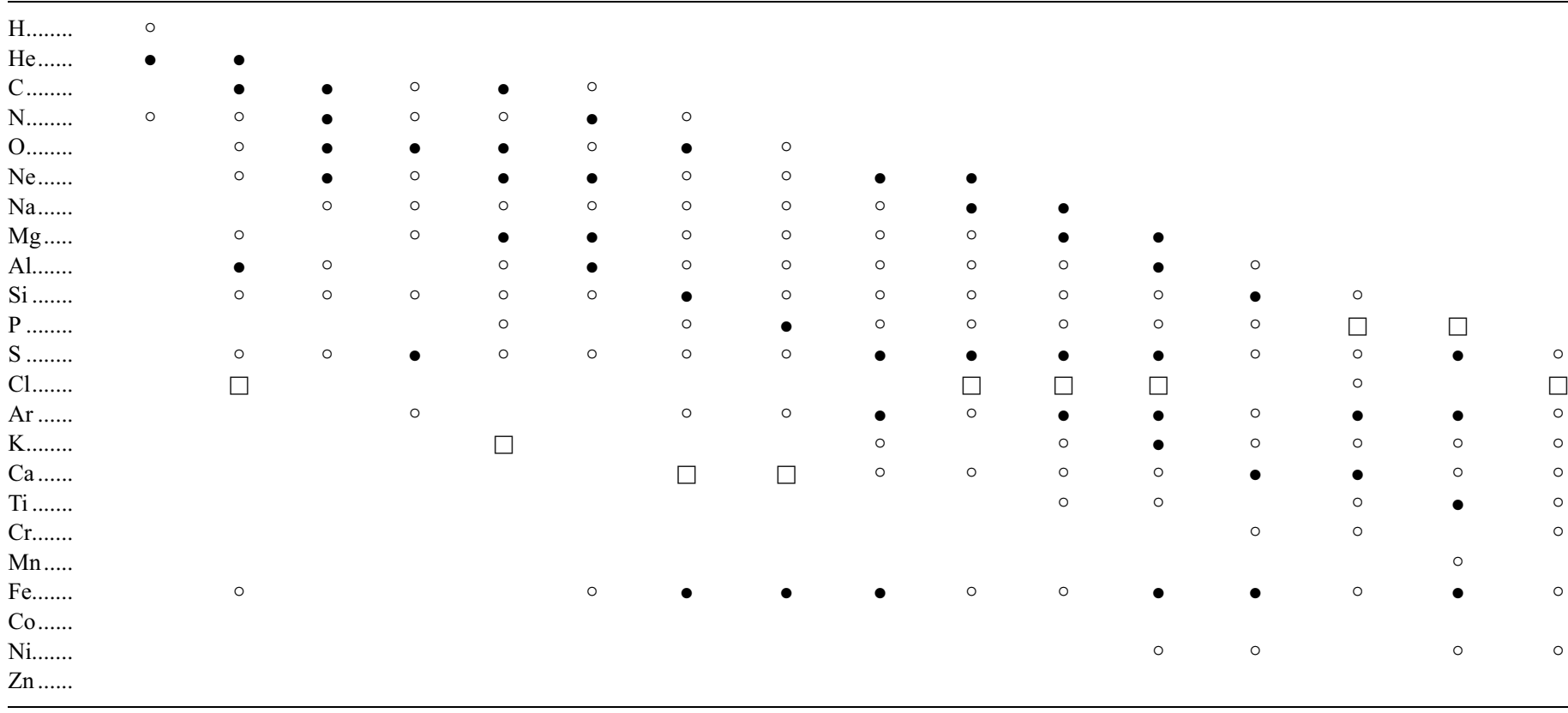

Notes.-( ${ }^{\circ}$ Ions in CHIANTI version 4.2 not changed in the present version. $(\bullet)$ Ions in the CHIANTI version 4.2 whose data have been modified/complemented in the present version. ( $\square$ ) New entries for the CHIANTI database.

(Kashyap \& Drake 2002), and the Arcetri Spectral Code (Landi \& Landini 2002). Also, CHIANTI has been included in the standard analysis software in a number of satellite missions such as SOHO EIT, SOHO CDS, TRACE, RHESSI, and RESIK, and will be used for future satellites such as SECCHI, Solar-B, and the Solar Dynamics Observatory (SDO).

Since the beginning of the CHIANTI project, the database has been continually expanded and updated to include new, more accurate calculations that appeared in the literature. In many cases, when no data were available in the literature, we have collaborated with atomic physicists to produce the data ourselves, so the CHIANTI project has been the driver of many calculations that have improved existing data sets or have provided the only data sets available for several ions or individual configurations within many different atomic systems.

Since the first release in 1996, new versions of CHIANTI have been released in 1999 (ver. 2; Landi et al. 1999), 2001 (ver. 3; Dere et al. 2001), and 2003 (ver. 4; Young et al. 2003). Each new version included new data, expanded the database to new ions, and included new physical processes. Initially, CHIANTI was designed to include data (energy levels, oscillator strengths, $A$-values, and collisional excitation rates) to calculate line intensities from collisionally excited plasmas, but new processes have been included in subsequent releases of CHIANTI that contribute to the line formation, such as proton excitation rates and photoexcitation from background blackbody radiation. The new version of CHIANTI described in the present paper follows the same pattern and has been developed in order to expand and improve the database in the X-ray range below $20 \AA$, in order to meet the needs of the most recent instruments such as XMM-Newton, Chandra, and Astro-E2. Important improvements have also been made for all other wavelength ranges, up to $2000 \AA$.
The new physical processes introduced in CHIANTI are ionization and recombination as population mechanisms of individual fine-structure levels, photoexcitation from a user-defined radiation field, and software to calculate CHIANTI spectra in cases when the distribution of velocities of free electrons is not Maxwellian.

New data for many ions have been published for the first time in the literature since the last release of CHIANTI, and they have been included in the present release. These data allow the calculation of emissivities of lines emitted by levels in high-energy configurations in Fe ions Fe XVII to Fe XXIII, in the 7-18 $\AA$ X-ray wavelength range, and of lines emitted in the UV range between 900 and $2000 \AA$ by transitions between excited configurations with principal quantum numbers $n=3$ in C-like, N-like, and O-like ions. Additional data for ions not included in the previous versions of the database have also been added. The data set for the K-shell emission from iron ions has been greatly expanded with new, accurate calculations, and a thorough review of energy levels and line identifications in the 7-18 A wavelength range has been performed.

The data for a few crucial ions already present in CHIANTI have been completely renewed and greatly improved and expanded. The most important of these ions are Fe IX, Fe XII, and Fe xv, which emit some of the strongest lines in the 170-450 spectral range; intensities from lines emitted by these ions are the main contributors to the total emission observed by narrowband imagers on board current and future NASA missions such as SOHO, SECCHI, and SDO.

A list of the ions included in the present version of CHIANTI and of the changes from the previous version for each individual ion can be found in Tables 1 and 2 .

The present paper describes the new processes inserted in the computation of CHIANTI emissivities in $\S 2$, while the new data 
TABLE 2

Ions Included in the CHIANTI Database

\begin{tabular}{|c|c|c|c|c|c|c|c|c|c|c|c|c|}
\hline Ion & XVII & XVIII & $\mathrm{XIX}$ & $\mathrm{xx}$ & XXI & XXII & XXIII & XXIV & $\mathrm{XXV}$ & XXVI & XXVII & XXVIII \\
\hline $\mathrm{Cl} \ldots \ldots \ldots \ldots$ & $\square$ & & & & & & & & & & & \\
\hline $\operatorname{Ar} \ldots \ldots \ldots \ldots$ & $\bullet$ & $\circ$ & & & & & & & & & & \\
\hline К & $\circ$ & $\square$ & $\square$ & & & & & & & & & \\
\hline $\mathrm{Ca} \ldots \ldots \ldots \ldots$ & $\circ$ & $\circ$ & • & $\circ$ & & & & & & & & \\
\hline $\mathrm{Ti}$ & $\circ$ & $\circ$ & $\circ$ & $\circ$ & & & & & & & & \\
\hline $\mathrm{Cr} . \ldots \ldots \ldots \ldots$ & $\bullet$ & $\circ$ & $\circ$ & $\circ$ & $\circ$ & $\circ$ & & & & & & \\
\hline $\mathrm{Mn} \ldots \ldots \ldots \ldots \ldots$ & $\circ$ & - & $\circ$ & $\circ$ & $\circ$ & $\circ$ & ○ & & & & & \\
\hline $\mathrm{Fe}$ & • & - & - & - & - & - & • & - & • & $\circ$ & & \\
\hline Сo................... & ○ & & $\circ$ & $\square$ & $\circ$ & $\circ$ & $\circ$ & $\circ$ & $\circ$ & & & \\
\hline Ni.................... & $\circ$ & $\circ$ & $\circ$ & $\circ$ & - & $\circ$ & $\circ$ & $\circ$ & $\circ$ & $\circ$ & $\circ$ & $\circ$ \\
\hline $\mathrm{Zn}$ & & & & $\circ$ & & & $\square$ & $\circ$ & $\circ$ & & $\circ$ & $\circ$ \\
\hline
\end{tabular}

Notes.-( $($ ) Ions in CHIANTI version 4.2 not changed in the present update. (•) Ions in CHIANTI version 4.2 whose data have been modified/ complemented in the present update. ( $\square$ ) New entries for the CHIANTI database.

for K-shell emission is presented in $\S 3$ and the energy level assessment is described in $\S 4$. The new data for the standard database are described in $\S 5$.

\section{NEW PROCESSES IN CHIANTI}

\subsection{Multitemperature Particle Distributions}

Within CHIANTI the assumption of Maxwellian electron and proton distributions is implicit through the storage of Maxwellianaveraged electron and proton collision strengths in the .SPLUPS and .PSPLUPS data files. To model emission from plasmas with general, non-Maxwellian particle distributions would require integrations of the original collision strengths with the new particle distributions, and this is outside the scope of the CHIANTI database.

However, if the particle distributions can be expressed as a linear combination of Maxwellians of different temperatures, i.e.,

$$
f\left(E ; a_{i}\right)=\sum_{i} a_{i} f_{\mathrm{M}}\left(E, T_{i}\right)
$$

where the Maxwellian function $f_{\mathrm{M}}$ is given by

$$
f_{\mathrm{M}}(E, T)=2\left(\frac{E}{\pi}\right)^{1 / 2}\left(\frac{1}{k T}\right)^{3 / 2} \exp \left(-\frac{E}{k T}\right),
$$

then such distributions can be modeled in a straightforward manner within the CHIANTI framework.

The generalized electron excitation rate coefficient for the transition $j$ to $k$ and for the particle distribution $f$ is given by

$$
\begin{aligned}
\mathcal{C}_{j k} & =\int_{E_{j k}}^{\infty} Q_{j k} v f\left(E ; a_{i}\right) d E \\
& =\sum_{i} a_{i} \int_{E_{j k}}^{\infty} Q_{j k} v f_{\mathrm{M}}\left(E, T_{i}\right) d E \\
& =\sum_{i} a_{i} C_{j k}\left(T_{i}\right)
\end{aligned}
$$

where $E_{j k}$ is the threshold energy for the transition, $Q_{j k}$ is the collision cross section, $E\left(=m_{e} v^{2} / 2, m_{e}\right.$ being the electron mass) is the energy of the incoming electron, and $C_{j k}\left(T_{i}\right)$ is the electron excitation rate coefficient for a Maxwellian particle distribution of temperature $T_{i}$ (see, e.g., Burgess \& Tully 1992).

The matrix $\mathcal{C}_{j k}$ replaces the usual Maxwellian-derived rate coefficient $\left(C_{j k}\right)$ in the level balance equations solved by the CHIANTI software. The software routines for calculating emissivities and level populations have been modified to allow input of the multitemperature parameters $a_{i}$ through the keyword SUM_MWL_ COEFFS. The temperatures $T_{i}$ are specified through the standard temperature input to the routines. The temperatures are assumed to apply to both proton and electron distributions and can take any values, with no limit to the number of temperatures included.

This prescription for treating non-Maxwellian distributions is not compatible with the treatment of ionization and recombination described in $\S 2.3$ since ion fractions defined at a single temperature are required for computing the correction factor to level populations due to these two processes, and the correction factor itself is only applied after the level balance equations have been solved. In such cases the ionization and recombination processes are switched off when calculating the level populations if the $a_{i}$ coefficients are specified in CHIANTI.

Table 3 demonstrates the effects of non-Maxwellian distributions on two key line ratios of $\mathrm{O}$ VI. The $\lambda 1032, \lambda 173$, and $\lambda 150$ lines are the strongest transitions of the $2 s-2 p, 2 p-3 d$, and $2 s-3 p$ multiplets, respectively, and we consider distributions composed of Maxwellians at $\log T=5.5$ and $\log T=6.0$. Varying the parameters $a_{i}$ as indicated in the table yields significant differences in the ratios as the higher energy electrons of the $\log T=6.0$ distribution are better able to excite the high-lying $n=3$ levels.

\subsection{Photoexcitation by Arbitrary Radiation Fields}

Version 4 of CHIANTI introduced the possibility of including photoexcitation and stimulated emission through an external blackbody radiation field into the level balance equations. With version 5 the software has been modified to allow an arbitrary, user-defined radiation field to be specified.

The user must create an IDL routine that calculates the energy density per unit wavelength, $U_{\lambda}$, as a function of wavelength.

TABLE 3

Non-Maxwellian Effects on O vi Line Ratios

\begin{tabular}{ccccc}
\hline \hline \multirow{2}{*}{ LINE RATIO } & \multicolumn{4}{c}{$\left[a_{1}, a_{2}\right]$} \\
\cline { 2 - 5 } & {$[1.0,0.0]$} & {$[0.75,0.25]$} & {$[0.5,0.5]$} & {$[0.0,1.0]$} \\
\hline$\lambda 150 / \lambda 1032 \ldots \ldots \ldots \ldots . .$. & 0.013 & 0.030 & 0.047 & 0.084 \\
$\lambda 173 / \lambda 1032 \ldots \ldots \ldots \ldots . .$. & 0.020 & 0.044 & 0.068 & 0.119
\end{tabular}




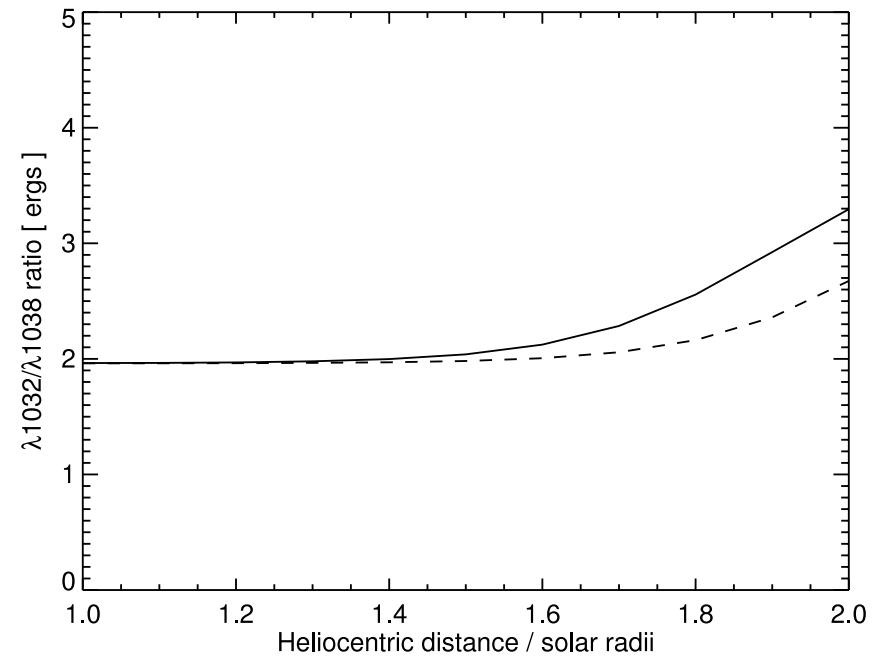

FIG. 1.-Variation of the O vI $\lambda 1032 / \lambda 1038$ ratio with distance from the Sun, assuming a uniform decrease of the electron density with height and that the emitting ions are illuminated by the average quiet Sun fluxes of the two lines (see text for more details). The solid line assumes no velocity separation between the emitting ions and solar surface; the dashed line is for the emitting ions moving away from the solar surface at a velocity of $40 \mathrm{~km} \mathrm{~s}^{-1}$.

The photoexcitation rate for a transition $i \rightarrow j$ is related to $U_{\lambda}$ by the expression

$$
P_{i j}=A_{j i} W(R) \frac{\omega_{j}}{\omega_{i}} \frac{\lambda^{5}}{8 \pi h c} U_{\lambda},
$$

where $A_{j i}$ is the radiative decay rate for the transition, $W(R)$ is the radiative dilution factor (see, e.g., Young et al. 2003), $\omega_{j}$ and $\omega_{i}$ are the statistical weights of the level $j$ and $i$, respectively, $h$ is Planck's constant, and $c$ is the speed of light. For reference we note that the energy density is related to the specific intensity by $I_{\lambda}=c U_{\lambda} / 4 \pi$.

The user-defined radiation field function is implemented through a keyword RADFUNC=' $u$ user_function, $a, b$ ' in the CHIANTI IDL routines SHOW_POPS and EMISS_CALC. The IDL routine USER_FUNCTION needs to be constructed simply to return a value for $U_{\lambda}$ for any input wavelength. The optional coefficients $\mathrm{a}$ and $\mathrm{b}$ can be used to modify the radiation field, e.g., by specifying a relative velocity between the radiation field and incident ion. Examples of possible functions that could be used are stellar atmosphere model spectra, power-law spectra, and bremsstrahlung spectra. More details can be found in the CHIANTI user guide available in the CHIANTI Web pages (see $\S 7$ ).

An example of the use of the RADFUNC keyword is demonstrated in Figure 1. Measurements of the $O$ VI $\lambda 1032 / \lambda 1038$ ratio at heights $1-10 R_{\odot}$ above the solar surface show variations from the 2:1 ratio expected when the transitions are electron excited (see, e.g., Fig. 17 of Kohl et al. 1997). This is due to $\mathrm{O}$ vi photons from the Sun's transition region becoming a significant excitation source in the low-density regions of the outer solar corona. This effect can now be modeled with CHIANTI by specifying RADFUNC to be a spectrum consisting of two Gaussians at the locations of the two $\mathrm{O}$ VI resonance lines. The RPHOT keyword (see $\S 2.4$ of Young et al. 2003) is used to specify distance from the radiation source. The intensities are assumed to be average quiet Sun values (Vernazza \& Reeves 1978) and their widths taken to be $0.2 \AA$. Choosing the electron density to fall uniformly with height from $10^{10} \mathrm{~cm}^{-3}$ at the solar surface to $10^{6} \mathrm{~cm}^{-3}$ at $1 R_{\odot}$ above the surface yields the $\lambda 1032 / \lambda 1038$ ratio shown in Figure 1. Specifying an outflow velocity of $40 \mathrm{~km} \mathrm{~s}^{-1}$ reduces the effects of photoexcitation as the exciting photons are redshifted relative to the outflowing ions and so less able to excite the transitions (this process is referred to as Doppler dimming).

\subsection{Ionization and Recombination}

Ionization and recombination processes were recognized to be of great importance for the calculation of line emissivities in the X-ray range by Doron \& Behar (2002) and Gu (2003). The former work calculated $\mathrm{Fe}$ XVII emissivities using a three-ion approach and demonstrated that contributions from dielectronic recombination, as well as resonant excitation, are of crucial importance to the level populations of Fe XVII. Gu (2003) extended this result to all Fe ions from Fe XVII to Fe XXIII by showing that all these ions are in many cases strongly influenced by direct recombination or cascades following recombination from higher levels. Such influences are directly translated into changes in the line emissivities.

In version 5 of CHIANTI, we have included ionization and recombination into level populations. The CHIANTI model for ionization and recombination assumes that the plasma can be described under the coronal model approximation, where the total population of the excited levels of an ion is negligible compared to the population of the ground level. In this case, recombination and ionization processes can be included in a relatively straightforward way, since they can be treated as a correction to the case where populations are calculated neglecting them.

To illustrate this method, we will consider the simplified atomic model of an ion $X^{+q}$ with abundance $n_{q}$ composed of the ground level and one excited level only. In case ionization and recombination contributions to level populations are negligible, the relative population of the upper level is obtained by solving the equation

$$
N_{g} N_{e} C_{g \rightarrow i}=N_{i} A_{i \rightarrow g} \Rightarrow\left(\frac{N_{i}}{N_{g}}\right)_{\text {no ion } / \mathrm{rec}}=\frac{N_{e} C_{g \rightarrow i}}{A_{i \rightarrow g}},
$$

where $C_{g \rightarrow i}$ is the collisional excitation rate and $A_{i \rightarrow g}$ is the Einstein coefficient for spontaneous radiative decay. Collisional de-excitation is neglected in the coronal model approximation. In case ionization and recombination provide significant contribution, equation (7) needs to be modified to include the rate coefficients for ionization $\left(\alpha_{\text {ion }}\right)$ and recombination $\left(\alpha_{\text {rec }}\right)$ :

$$
N_{g} N_{e}\left(n_{q} C_{g \rightarrow i}+n_{q-1} \alpha_{\text {ion }}+n_{q+1} \alpha_{\text {rec }}\right)=N_{i} A_{i \rightarrow g} n_{q},
$$

where $n_{q-1}, n_{q}, n_{q+1}$ are the ion fractions for the ions $X^{q-1}$, $X^{q}$, and $X^{q+1}$, respectively. Here $\alpha_{\text {ion }}$ and $\alpha_{\text {rec }}$ are the effective ionization and recombination rates, which take into account both direct ionization and recombination to each excited level, and cascade contributions from higher levels ( Gu 2003). The population of the excited level can then be expressed as

$$
\left(\frac{N_{i}}{N_{g}}\right)_{\mathrm{ion} / \mathrm{rec}}=\left(\frac{N_{i}}{N_{g}}\right)_{\text {no ion } / \mathrm{rec}} \times \aleph
$$

where the correction $\aleph$ is given by

$$
\aleph=1+\frac{n_{q-1} \alpha_{\text {ion }}+n_{q+1} \alpha_{\text {rec }}}{n_{q} C_{g \rightarrow i}} .
$$

The correction $\aleph$ is temperature sensitive and can be large when the collisional excitation rate is small or when the abundance 


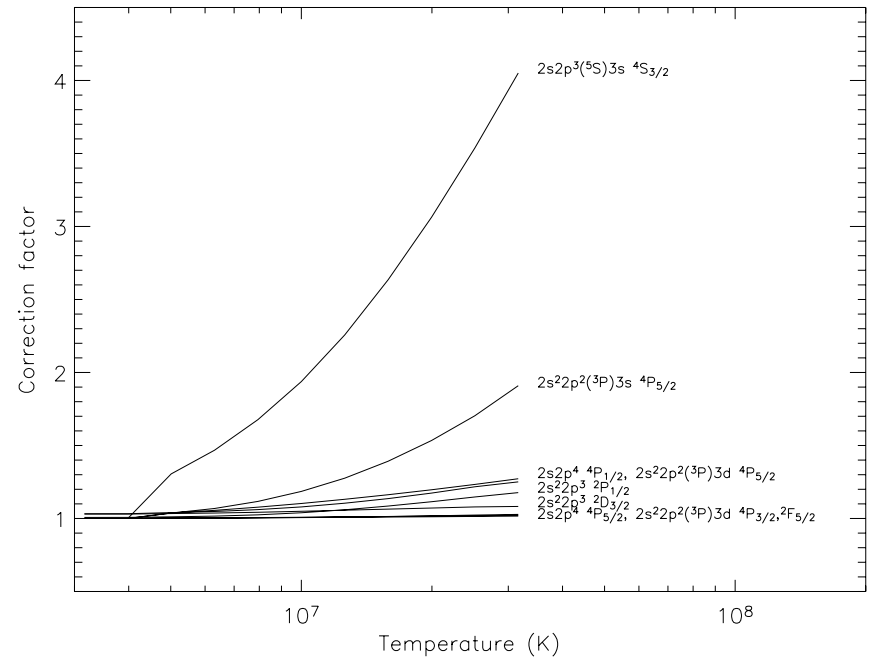

FIG. 2.-Correction factor $\aleph$ as a function of temperature for the Fe xx levels that give rise to observed transitions in the Fe xx spectrum. The Fe xx maximum abundance temperature is $T \simeq 9 \times 10^{6} \mathrm{~K}$.

of the ion $q$ is much smaller than the abundances of the adjacent ions. The correction due to ionization and recombination can have significant effects on intensities of observed X-ray lines. Examples are given in Figure 2, where the value of $\aleph$ is displayed as a function of temperature for selected levels of Fe XX giving rise to observed lines. In general the largest correction factors are found for levels with low collisional excitation rates, whose level populations are low and therefore do not give rise to observed lines. However, the emissivity of several observed lines changes significantly, although for them the correction is normally less than a factor 2 .

The only limitation of this approach lies in the breakdown of the coronal model approximation at high densities for a few ions. In particular, the greater problems arise for $\mathrm{Fe} \mathrm{Xx}$ and $\mathrm{Fe} \mathrm{XXI}$ when densities reach $10^{12} \mathrm{~cm}^{-3}$, since by then the populations of the metastable levels are significant and invalidate equations (1) and (2). However, such densities are reached only in strong flares. The other ions either start to have problems at $10^{13} \mathrm{~cm}^{-3}$ (Fe XxI) or higher, or can always be described by the coronal model approximation (Fe XXIV). The critical densities $N_{e}^{\max }$ above which metastable level populations begin to be nonnegligible are listed in Table 4. However, it is possible that the effects of metastable levels on predicted line emissivities start to be nonnegligible at higher densities than reported in Table 4.

We have included ionization and recombination in CHIANTI for the highly ionized Fe ions (Fe XVII-XXIII) and for the ions of the most abundant elements in the hydrogen and helium isoelectronic sequences. The CHIANTI software has been modified to allow calculation of the correction factor $\aleph$ for the ions for which $\alpha_{\text {ion }}$ and $\alpha_{\text {rec }}$ are provided.

We created two new files that include the $\alpha_{\text {ion }}$ and $\alpha_{\text {rec }}$ rates. The names of these files follow the usual CHIANTI pattern and have the .CILVL and .RECLVL suffixes, for ionization and recombination, respectively. Data in these files are tabulated as a function of temperature, at all temperatures for which they are provided.

\subsubsection{Hydrogen and Helium Isoelectronic Sequences}

Recombination rates for the $\mathrm{H}$-like and He-like ions are provided for the most abundant elements in the universe. These are C, N, O, Ne, Na, Mg, Al, Si, S, Ar, Ca, Fe, and Ni. For these elements, we have included data for radiative recombination. In
TABLE 4

Minimum Densities

\begin{tabular}{|c|c|}
\hline Ion & $\log N_{e}^{\min }$ \\
\hline H-like ions ...... & Any density \\
\hline 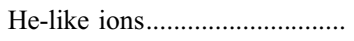 & Any density \\
\hline Fe XXVII & $>15$ \\
\hline Fe XVIII. & 13.5 \\
\hline Fe xIx & 13 \\
\hline Fe xx & 12.5 \\
\hline 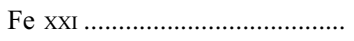 & 12 \\
\hline 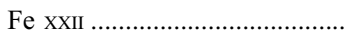 & 13 \\
\hline 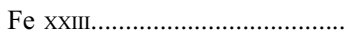 & $>15$ \\
\hline Fe xxIV & Any density \\
\hline
\end{tabular}

Notes.-Minimum densities at which metastable levels are no more negligible in the ions for which ionization and recombination rates are available. Metastable populations are considered nonnegligible when the population of at least one metastable level reaches $10 \%$ of the total population of the ion.

future versions of CHIANTI, we will include also the effects of dielectronic recombination for He-like ions.

The recombination rates have been calculated using the formulae and the constants provided by Mewe et al. (1985). The radiative recombination coefficient is given by

$$
\alpha_{\mathrm{RR}}=1 \times 10^{-11}(z+1)^{2 \eta+1} a_{\mathrm{rec}} T^{-\eta},
$$

where $z$ is the stage of ionization of the ion we are considering, and the parameters $\eta$ and $a_{\text {rec }}$ are taken from the tabulations published by Mewe et al. (1985). These parameters are given for the H-like $n p^{2} P$ doublets with $n=2-5$ and for the He-like levels $1 s 2 p{ }^{3} P_{1,2}, 1 s 2 s^{3} S_{1}$, and 1 snp ${ }^{1} P_{1}$ with $n=2-5$.

\subsubsection{Ca XIX and Fe XXv}

The only He-like elements to be treated separately from the rest of the sequence are $\mathrm{Ca}$ XIX and Fe XXV. For these two ions, level-resolved recombination rates for the $n=2$ levels in Ca XIX and Fe Xxv were published by Bely-Dubau et al. (1982b) and Bely-Dubau et al. (1982a), respectively.

In both works, total recombination rates were presented for the levels giving rise to the four bright lines emitted by the $n=2$ complex: $1 s 2 s{ }^{3} S_{1}, 1 s 2 p{ }^{1} P_{1}$, and $1 s 2 p^{3} P_{1,2}$. The total rates are the sum of the radiative recombination and dielectronic recombination rates. Radiative recombination rates for the 1 snp ${ }^{1} P_{1}$ levels $(n=3,4,5)$ have been calculated using equation (11).

\subsubsection{Fe XVII to Fe XXIII}

Rates for ionization and recombination to excited levels in the $n=2$ and $n=3$ configurations of Fe XVII to Fe XXIII are provided by $\mathrm{Gu}$ (2003). Gu (2003) was the first to present a systematic study of the role of indirect processes in the soft X-ray line formation of Fe L-shell ions, demonstrating that ionization and recombination were important for populating individual finestructure levels in Fe XVII to Fe XXIV.

$\mathrm{Gu}$ (2003) provides the rate coefficients necessary to calculate the correction factor $\aleph$ for all the levels in the $n=2$ and $n=3$ complexes. These rate coefficients include collisional ionization, radiative recombination, and dielectronic recombination, taking into account the contributions of cascades. All these data have been included in the present version of CHIANTI.

$\mathrm{Gu}$ (2003) has shown that dielectronic and radiative recombination are even more important to level populations than 
resonances at low temperatures and are higher than, or comparable to, the latter at all temperature regimes. Collisional ionization is nonnegligible only for Fe XVII at high energies.

\section{K-SHELL EMISSION IN Fe}

Spectral lines emitted by K-states in Fe dominate the soft X-ray spectrum below $2 \AA$. These lines are originated from K-vacancy states, where an electron is missing in the $1 s$ shell, and they have been observed in almost all X-ray astronomical sources, as well as in the laboratory. The diagnostic potential of these lines is well known, and it has been used to measure the physical properties of high temperature plasmas.

K-shell emission in Fe XXv (He-like sequence) and Fe XXVI (H-like sequence) was already included in earlier CHIANTI versions, and treated using a normal collisional-radiative model like all other ions in the database since the electronic structure of H-like and He-like includes an open $1 s$ shell.

Fe ions with more than two electrons have a closed $1 s$ shell, so K-vacancy states lie above the ionization threshold and generate satellite lines to strong resonance lines. These levels can be excited by several main processes:

1. direct collisional excitation: $X_{j}^{q}+e\left(E_{1}\right) \rightarrow X_{k}^{q}+e\left(E_{2}\right)$, with $E_{2}<E_{1}$

2. photoexcitation from X-ray radiation: $X_{j}^{q}+h \nu \rightarrow X_{k}^{q}$;

3. photoionization from X-ray radiation: $X_{j}^{q-1}+h \nu \rightarrow$ $X_{k}^{q}+e$

4. radiationless capture of a free electron into a bound excited state above the ionization threshold $X_{j}^{q}+e \rightarrow X_{k}^{q-1}$; and

5. charge exchange between neutral gases and highly charged ions: $X_{j}^{q}+A \rightarrow X_{k}^{q-1}+A^{+}$,

where $j$ is the initial state of the ion $X^{q}, k$ is the state of the ion $X^{q}$ or $X^{q-1}$ with energy above the ionization threshold, $e(E)$ is the incident electron with kinetic energy $E$, and $h \nu$ indicates the photoexciting photon with energy $h \nu$ and frequency $\nu$.

Once the ion is in this highly excited state, there are two main avenues for de-excitation: spontaneous radiative decay to levels with energy lower than the ionization threshold, or autoionization. In the first case, an X-ray photon is emitted; in the second case, the ion loses an electron but does not emit any radiation.

In CHIANTI we are interested in the lines generated by the radiative decay of the highly ionized state. This line emission can be grouped into two classes of transitions ( $\mathrm{K} \alpha$ and $\mathrm{K} \beta$ ), according to the final configurations that are involved in the transition. $\mathrm{K} \alpha$ emission is generated when the final state is a configuration with a vacancy in the $n=2$ shell (i.e., Fe Xv $1 s^{2} 2 s^{2} 2 p^{5} 3 s^{2} 3 p$ ). $\mathrm{K} \beta$ emission arises when the final configuration has both the $n=$ 1 and $n=2$ shells filled, a vacancy in the $n=3$ shell, and energy below the ionization threshold. $\mathrm{K} \beta$ lines are found at shorter wavelengths than $\mathrm{K} \alpha$ lines.

The wavelengths of $\mathrm{K} \alpha$ and $\mathrm{K} \beta$ lines vary between 1.7 and $1.9 \AA$, but for $\mathrm{Fe}$ II to $\mathrm{Fe}$ XVI they are grouped in very narrow ranges, $1.72-1.76 \AA$ for $\mathrm{K} \beta$, and 1.925 and $1.942 \AA$ for $\mathrm{K} \alpha$. They are not resolved easily by existing spectrometers, and they form two distinct spectral features.

In the present version of CHIANTI, we have included atomic data and transition rates for $\mathrm{K} \alpha$ and $\mathrm{K} \beta$ lines for $\mathrm{Fe}$ II to $\mathrm{Fe}$ XXIV. Data for Fe XXV and Fe XXVI were already in the previous versions of CHIANTI. As excitation mechanisms, we have included dielectronic capture of a free electron (dielectronic excitation) for all ions, and direct collisional excitation (inner-shell excitation) for Fe XVIII to Fe xxIV. Other excitation processes for K-states are not yet included in CHIANTI. The data necessary to include photoexcitation are available, but will be included in the next release of CHIANTI.

\subsection{Inner-Shell Excitation}

Inner-shell excitation of K-shell states was already included in earlier versions of CHIANTI only for Fe XXIV and Fe XXIII. However, the Fe XXIII model included unpublished data for only two transitions obtained with the Flexible Atomic Code (FAC; Gu 2003), while Fe XXIV data consisted of old calculations from Goett \& Sampson (1983). In the present version of CHIANTI we include new, complete, and accurate data for Fe XVIII to Fe XXIV.

The treatment of inner-shell excitation in CHIANTI is straightforward, since the K-shell levels and their collisional excitation can be included in the same way as any other levels in the database. The only difference lies in the depopulation of these levels, which can be achieved both by radiative decay and autoionization. In order to take into account the latter process, autoionization rates are included in the .WGFA files of each of these ions and added to the total depopulation rate in the equations for statistical balance. Details can be found in Dere et al. (2001).

\subsubsection{Fe XXIV}

Inner-shell excitation for K-states in Fe XxIv has been included for all the levels in the configurations $1 s 2 s^{2}, 1 s 2 s 2 p$, and $1 s 2 p^{2}$. The energies for these levels have been taken from the calculations of Bautista et al. (2003). Autoionization rates and $A$-values for all the important transitions between these levels are taken from Palmeri et al. (2003a). Also Bautista et al. (2003) provide these rates, but for the sake of consistency with the autoionization values in the dielectronic excitation files (see $\S 3.2$ ), we have retained the data from the former calculation. Differences between the two sets of data are negligible. Wavelengths for these transitions are taken from the calculated values, and their accuracy was found to be of $\approx 0.3-0.6 \mathrm{~m} \AA$.

Electron excitation rates for K-states have been taken from Bautista et al. (2003). They carried out a very detailed calculation of effective collision strengths by using a target representation that includes all the 19 levels in the $n=2$ complex and the BreitPauli $\boldsymbol{R}$-matrix package, including one-body relativistic corrections. The importance of the $n=3$ target states, convergence of partial-wave expansion, two-body relativistic corrections, and Auger and radiative dampings were investigated extensively, finding that dampings have a crucial importance in reducing the importance of resonances; the other effects were negligible and convergence was achieved satisfactorily. Bautista et al. (2003) provide Maxwellian-averaged collision strengths for temperatures in the $10^{5}-10^{8} \mathrm{~K}$ range, plus an asymptotic value at very high temperature.

It is important to note that Whiteford et al. (2002) provide innershell collisional excitation rates for the $n=2$ and $n=3$ levels above the ionization threshold. However, they do not provide the autoionization rates, so data for the $n=3$ levels above ionization could not be included in the present version of CHIANTI.

\subsubsection{Fe XVIII-XXIII}

Energy levels, wavelengths, and radiative data and Auger rates for all the levels in the $1 s 2 s^{p} 2 p^{k}$ complexes of these ions are taken from Palmeri et al. (2003a). Calculated level energies and wavelengths are treated as experimental values in the .WGFA files; Palmeri et al. (2003a) assessed their accuracy against observed and theoretical values in the literature and found that accuracies of the wavelengths were within $2 \mathrm{~m} \AA$. 
Maxwellian-averaged collision strengths are taken from the extensive calculations of Bautista et al. (2004). These authors included all the levels considered by Palmeri et al. (2003a) and calculated a complete set of energy levels, $A$-values, Auger rates, and Maxwellian-averaged collision strengths for all levels and transitions involving them.

Bautista et al. (2004) make use of the Breit-Pauli $\boldsymbol{R}$-matrix package and include the effects of radiation and Auger dampings in their calculations; these effects practically wash out the resonance structure in the collision strengths. Convergence at high energy is also given special attention.

\subsection{Dielectronic Recombination}

Data for the calculation of dielectronic satellite lines for $\mathrm{Fe}$ ions were included in the previous version of the CHIANTI database for Fe xxI to Fe xxv. The data were taken from calculations performed by Safronova and reported by Kato et al. (1997). In the present version of CHIANTI, we have replaced all of these data, with the only exception of Fe xxv, with the more recent calculations of M. Bautista and coworkers. These new calculations have been carried out with state-of-the-art computer codes after a thorough comparison between different codes and approximations and laboratory observations. Also, these calculations have been performed for all $\mathrm{Fe}$ ions from $\mathrm{Fe}$ II to $\mathrm{Fe}$ xxIv.

The collisional excitation rate $C_{d}^{j k}$ of a line produced by dielectronic recombination can be expressed using detailed balance considerations (Gabriel \& Paget 1972; Doschek 1985) as

$$
C_{d}^{j k}=\frac{h^{3}}{2\left(2 \pi m_{e} k T\right)^{3 / 2}} \frac{g_{u}}{g_{l}} A_{a}^{j k} e^{-\Delta E / k T}
$$

where $j$ is the initial level of the ion $X^{q}, k$ is the level of the ion $X^{q-1}$ whose energy is above the ionization threshold of $X^{q-1}$, $A_{a}^{j k}$ is the partial autoionization rate from level $k$ back to level $j$, $h$ and $k$ are the Planck and Boltzmann constants, $m_{e}$ is the mass of the electron, $g_{u}$ and $g_{l}$ are the upper and lower level statistical weights, and $\Delta E$ is the energy difference between the $k$ and $j$ levels.

Equation (12) shows that for ions formed at temperatures for which $\Delta E / k T \simeq 1$, collisional excitation is an efficient means of level population; such is the case for Fe xxI to Fe Xxv ions, whose formation temperatures are of the order of $10 \mathrm{MK}$. For ions formed at lower temperatures, the exponential term decreases very fast, so that dielectronic capture loses importance as an excitation mechanism. For these ions, K-shell level population is mainly driven by other processes, some of which are not yet included in CHIANTI, such as photoexcitation and photoionization from X-ray radiation, or charge exchange.

\subsubsection{Fe xvIII to Fe xxIV}

Palmeri et al. (2003a) carried out extensive calculations of energy levels and radiative and Auger rates for K-vacancy states within the $n=2$ complex of Fe XVIII to Fe Xxv, and their data supersede the data already available in CHIANTI for all these ions. In the case of Fe xxv the transitions considered by Palmeri et al. (2003a) involve levels below the ionization threshold, so only Einstein coefficients for spontaneous radiative decay were provided and they are described in $\S$ 5.2.5. The data from Palmeri et al. (2003a) are the same as already described in $\S$ 3.1.2.

Fe xxIv data were further complemented with data for additional transitions between $1 s^{2} 2 l$ levels $(l=s, p)$ and the $1 s 2 \ln l^{\prime}$ levels (with $n=3,4,5 ; l^{\prime} \geq 3$ ), calculated by M. Cornille and reported in Kato et al. (1997). These transitions, not present in previous versions of CHIANTI, give rise to many strong satellite lines in the 1.48-1.60 ̊̊ wavelength range that are important contributors to the total intensity observed by high- and low-resolution $\mathrm{X}$-ray spectrometers.

$$
\text { 3.2.2. Fe } \mathrm{x} \text { to } \mathrm{Fe} \mathrm{x} \text { II }
$$

Mendoza et al. (2004) report extensive calculations of level energies, wavelengths, radiative, and Auger rates for $\mathrm{Fe}$ ions from $\mathrm{Fe} x$ to Fe xVII. They make use of three different atomic codes to estimate the importance of several effects and to assess the reliability of the results. They estimate predicted wavelengths to be accurate within $2 \mathrm{~m} \AA$, and their Auger and radiative rates to be accurate within $20 \%$.

Mendoza et al. (2004) include all the K-shell levels in the lowest energy configuration with an $n=1$ hole, that is, $1 s 2 s^{2} 2 p^{6} \mu$, where $\mu$ is the lowest outer electron configuration of each ion. They provide radiative rates for the emission of $\mathrm{K} \alpha$ and $\mathrm{K} \beta$ transitions. Unfortunately, no radiative rates are provided for the calculation of satellite emission from the configurations with an $n=2$ hole that provide the lower levels of the $\mathrm{K} \alpha$ transitions; such levels give rise to lines at around $15-20 \AA$.

\subsubsection{Fe II to $\mathrm{Fe}$ IX}

Palmeri et al. (2003b) carried out calculations of level energies, wavelengths, and radiative and Auger rates for the lowest $\mathrm{K}$-shell vacancy fine-structure levels populated by photoionization of the ground state of the parent ion. These data are now included in CHIANTI. Palmeri et al. (2003b) compared the results they obtained from two different codes with other calculations and measurements in the literature, and determine the accuracy of their results to be $\simeq 10 \%$.

Unfortunately, no radiative rates are provided for the calculation of satellite emission from the configuration with an $n=2$ vacancy, predicted to fall at around 15-20.

$$
\text { 3.2.4. S xIV, Ca XVIII }
$$

Radiative decay rates for a number of dielectronic satellite lines missing in the previous version of CHIANTI have been added to both ions. These transitions connect the $n=2$ levels below the ionization threshold with the $1 s 2 \operatorname{lnl}(l=s, p ; n=3,4,5)$ levels, giving rise to several intense spectral lines at around 4.0-4.4 $\AA$ (S XIV) and 2.5-2.8 $\AA$ (Ca XVIII). These radiative data are taken from M.Cornille's calculations reported in Kato et al. (1997).

$$
\text { 3.2.5. Si XII }
$$

The present model for dielectronic line emission in Si XII has been greatly expanded from the previous versions of CHIANTI, to include all the possible configurations $1 s^{2} n l$ and $1 s n l^{\prime} l^{\prime}$ $\left(n, n^{\prime}=2,3,4\right)$ for a total of 188 levels above the ionization threshold. These data are taken from the extensive calculations by Phillips et al. (2006) and allow us to predict a large number of dielectronic satellite lines in the 5.3-6.9 ̊ range. Calculations were carried out using the SUPERSTRUCTURE code (Eissner et al. 1974) and the AUTOLSJ code (Dubau \& Loulergue 1982), and were merged with the data already present in CHIANTI.

\section{ENERGY LEVEL ASSESSMENT}

Since the release of version 4 of the database, a few new studies have been carried out with the aim of measuring the wavelengths and energy levels of highly ionized ions. These studies were made in response to the need of accurate line identifications, in order to avoid confusion and mismatches in spectral codes and to provide new identifications. These studies have been carried out both using laboratory sources and using spectra of solar flares. 
Measurements in the laboratory were mostly carried out by the Lawrence Livermore group. They noted that all current spectral codes lack spectral lines for the high-energy configurations with principal quantum number $n=3$ or higher, and used an EBIT device to measure the most prominent spectral lines emitted by these configurations in the X-ray ranges. Their efforts were first concentrated on highly ionized Fe ( Fe XVIII to Fe XXIV), resulting in a fairly extensive line list that included $n=3$ and $n=4$ levels (Brown et al. 2002). A subsequent study measured spectral lines in the 60-140 Å range from Fe viI to Fe x (Lepson et al. 2002). Measurements for Ar IX to Ar XVI in the 20-50 $\AA$ range (Lepson et al. 2003), for S VII to $S$ XIV in the 20-75 range (Lepson et al. 2005), and for Fe XV to Fe XIX in the 13.5$17 \AA$ range (May et al. 2005) have also been carried out; measurements for Mg are under way (P. Beiersdorfer 2004, private communication).

Energy level assessments have also been done using observations of solar high-resolution spectra. Recently, as part of the CHIANTI project, such assessment have been carried out in the X-ray range (Landi \& Phillips 2005; Del Zanna et al. 2005; Del Zanna 2006) and in the EUV, UV, and visible ranges (Del Zanna et al. 2004; Del Zanna \& Mason 2005). In these studies, level identifications for several highly ionized Fe ions have been reassessed and improved by using both line wavelengths and fluxes. The use of line fluxes from sophisticated spectral codes and large-scale atomic physics calculations is of great help in correctly assigning atomic transitions to each spectral feature, as well as assessing the presence of previously unidentified blending lines. This approach is a great improvement compared to many earlier studies, in which most energy level assignments were determined by using wavelengths, oscillator strengths, and the presence of the same transition emitted by other ions of the same isoelectronic sequence at different wavelengths.

The new laboratory measurements and the assessment of line and level identifications have been incorporated in version 5 of CHIANTI. The new assessments that we have carried out as part of the CHIANTI project allowed us to correct several misassignments in previous line lists, identify a number of new transitions, and improve greatly the completeness and the accuracy of CHIANTI spectral predictions. Many of the laboratory identifications from the Lawrence Livermore group were provided for the first time, so energies for many more levels than previously available have been included in CHIANTI.

\section{NEW DATA FOR THE STANDARD DATABASE}

\subsection{Hydrogen Isoelectronic Sequence}

\subsubsection{He II}

C. P. Ballance (2003, private communication) has performed an $\boldsymbol{R}$-matrix calculation, similar to that of Ballance et al. (2002), for He II for all fine-structure levels with $n \leq 5$. These collision strengths approach the correct high-energy limit (Burgess \& Tully 1992) and are used in the present version of CHIANTI.

$$
\text { 5.1.2. Ne } x
$$

A significant discrepancy in the important $\operatorname{Ly} \alpha / \operatorname{Ly} \beta$ ratio of $\mathrm{Ne} \mathrm{x}$ as calculated with CHIANTI and other codes has been pointed out (A. Maggio 2003, private communication). The ratio was overestimated by a factor of about 2 . The problem was due to an error in the tabulation of the collision strengths of Aggarwal \& Kingston (1991).

We have replaced the previous data with new data sets that now include 36 levels, up to $n=6$. Observed energies are from NIST version 3 (Ralchenko et al. 2005), while theoretical energies are from the new $\boldsymbol{R}$-matrix calculation of Ballance et al. (2003). $A$-values have been obtained from a standard SUPERSTRUCTURE calculation that included all levels up to $n=6$. We adopted the effective collision strengths calculated (in $L S$ coupling) by Ballance et al. (2003) in the temperature range $2 \times 10^{5}-2 \times 10^{7} \mathrm{~K}$. The fine-structure collision strengths have been obtained assuming a distribution according to statistical weights. Only excitations from the $1 s$ and $2 s$ levels are retained.

$$
\text { 5.1.3. Na XI }
$$

The CHIANTI model for $\mathrm{Na}$ XI data includes data interpolated using all the ions in the $\mathrm{H}$-like sequence, since no calculations are available in the literature. However, the errors found in the collision rates $\mathrm{Ne} x$ and $\mathrm{Mg}$ XII (see previous and next sections) have made a new interpolation of the $\mathrm{Na}$ XI collision rates necessary, and the results replace the older values available in the previous version of the database.

\subsubsection{Mg XII}

An error was found in the collisional data for the transition $2-5\left(2 s{ }^{2} S_{1 / 2}-3 s{ }^{2} S_{1 / 2}\right)$, for which the data of another transition were mistakenly used. This has now been corrected in version 5 .

$$
\text { 5.1.5. P xv, Cl xvII, K xIX }
$$

Spectral lines for these ions have been observed with the RESIK crystal spectrometer on board the Russian Coronas-F solar mission (Sylwester et al. 2004, 2005) and have been used to measure the abundances of potassium by Phillips et al. (2003). There are no data for these ions in the literature, so a complete data set of theoretical energy levels, $A$-values, oscillator strengths, and effective collision strengths has been interpolated using all the ions in the H-like sequence available in the CHIANTI database. The atomic model for these three ions includes 25 finestructure levels up to $n=5$. Experimental energies are taken from version 2 of the NIST database (Fuhr et al. 1999) and are available for all levels.

\subsubsection{Fe XXvI}

Ballance et al. (2002) have performed an $\boldsymbol{R}$-matrix calculation of the collisions strengths for all fine-structure levels with $n \leq 5$. They paid particular attention to the high-energy limit which, for allowed transitions, is given by the oscillator strength. Effective collision strengths are calculated in the $6.0 \leq \log T \leq 8.5$ temperature range; these values and theoretical energies provided by Ballance et al. (2002) replace the data previously available in CHIANTI. Radiative data have not been changed.

\subsection{Helium Isoelectronic Sequence}

$$
\text { 5.2.1. } \mathrm{P} \text { xIv, } \mathrm{Cl} \text { xvI, } \mathrm{K} \text { xviI }
$$

High-resolution X-ray crystal spectra from RESIK also include lines from P xIV, Cl XVI, and K XVII (Sylwester et al. 2004, 2005 ) that were not included in the earlier versions of CHIANTI due to the lack of transition rates for these ions in the literature. We have included them in the present version by interpolating all the necessary data (energy levels, $A$-values, oscillator strengths, and effective collision strengths) from the data for all the He-like ions included in CHIANTI. The atomic model includes data up to $n=5$ (49 fine-structure levels) for $\mathrm{Cl}$ XVI and $\mathrm{K}$ XVII, while it is limited to 45 fine-structure levels in the case of $\mathrm{P}$ XIV, since it was not possible to interpolate the data for the $1 \mathrm{~s} 5 \mathrm{~g}$ configuration accurately. Observed energy levels come from version 3 of 
TABLE 5

Papers with Laboratory Measurements of the $1 s^{2}{ }^{1} S_{0}-1 s 2 s{ }^{3} S_{1}$ and $1 s^{2}{ }^{1} S_{0}-1 s 2 p{ }^{3} P_{1}$ Transitions Used in CHIANTI

\begin{tabular}{|c|c|c|}
\hline \multirow[b]{2}{*}{ ION } & \multicolumn{2}{|c|}{ Transition } \\
\hline & ${ }^{1} S_{0}-{ }^{3} S_{1}$ & ${ }^{1} S_{0}-{ }^{3} P_{1}$ \\
\hline $\mathrm{C}$ v $\ldots \ldots \ldots \ldots \ldots$ & Schmidt et al. (1994) & Hutton et al. (1985) \\
\hline $\mathrm{N}$ VI .............. & Neill et al. (2000) & Hutton et al. (1985) \\
\hline $\mathrm{O}$ VII .............. & Crespo Lopez-Urrutia et al. (1998) & Engström et al. (1980) \\
\hline $\mathrm{Ne}$ IX........... & Träbert et al. (1999a) & .. \\
\hline Mg XI........ & Stefanelli et al. (1995) & Armour et al. (1981) \\
\hline $\mathrm{Si}$ XIII ......... & $\ldots$ & Armour et al. (1979) \\
\hline
\end{tabular}

the NIST database (Ralchenko et al. 2005), except for K xVII, where they are taken from Shirai et al. (2000).

5.2.2. He i, C v, N vi, O viI, Ne Ix, Mg XI, Si XIII, S xv, Ar xvII, Ca XIX

Slight deviations were found between the $A$-values and the weighted oscillator strengths $g f$ for several transitions between excited configurations from the formula

$$
g f=1.5 \times 10^{-16} \lambda^{2} A \omega
$$

where $\lambda$ is the transition wavelength and $\omega$ is the statistical weight of the upper level. These deviations were due to some confusion in the use of the wavelengths, but they never exceeded a few percent. Now this has been corrected.

$A$-values for a the two transitions $1 s^{2}{ }^{1} S_{0}-1 s 2 s^{3} S_{1}$ and $1 s^{2}{ }^{1} S_{0^{-}}$ $1 s 2 p^{3} P_{1}$ have also been changed for several ions, using the laboratory measurements as detailed in Table 5. Differences from the theoretical values previously available in CHIANTI are within a few percent.

\subsection{3. $\mathrm{Na} \mathrm{x}$}

Due to the lack of calculations in the literature, $\mathrm{Na} \times$ data in CHIANTI 4 were interpolated using data for all the other ions along the isoelectronic sequence. The changes made to the radiative rates of many ions of the sequence (see previous sections) have made a new interpolation necessary, and this has been done for CHIANTI 5. In addition, $A$-values for the transitions between the $1 s^{2}, 1 s 2 s$, and $1 s 2 p$ configurations have been taken from Lin et al. (1977).

\subsubsection{Al XII}

The collisional excitation rates from the ground level to the $1 s 2 s$ and $1 s 2 p$ configurations in CHIANTI 4, from Sampson et al. (1983) have been replaced with an interpolation of effective collision strengths available along the helium sequence from Zhang \& Sampson (1987). This change has been made in order to take into account resonances, included in the Zhang \& Sampson (1987) data, that were neglected in the data set available in CHIANTI 4. A few slight deviations between the $A$-values and the weighted oscillator strengths $g f$ for several transitions between excited configurations were also corrected, as described in $\S 5.2 .2$.

\subsubsection{Fe xxv}

The $A$-values from the extensive calculations by Palmeri et al. (2003a) replace older values from Lin et al. (1977) for radiative transitions from the $1 s 2 s$ and $1 s 2 p$ configurations. Differences between the two data sets are moderate. The two-photon rate for the $1 s 2 s{ }^{1} S_{0}-1 s 2 s{ }^{1} S_{0}$ transition and all other data for this ion are left unchanged.

\subsection{Lithium Isoelectronic Sequence}

\subsubsection{Fe XXIV}

The new data set consists of all levels up to $n=5$, for a total of 24 fine-structure levels. Additional data for the $n=2$ configurations above the ionization limit are also included, to take into account inner-shell transitions, as described in $\S 3.1$. Observed energies (and wavelengths) are from the benchmark study of Del Zanna (2006) and from version 3 of the NIST database (Ralchenko et al. 2005). The CHIANTI spline fits were obtained from the effective collision strengths calculated by Berrington \& Tully (1997) for the levels up to $n=4$, and by Whiteford et al. (2002) for the $n=5$ and the inner-shell levels. The rate coefficients are to be considered valid in the temperature range $1.6 \times 10^{6}-10^{8} \mathrm{~K}$.

Berrington \& Tully (1997) performed a full Breit-Pauli $\boldsymbol{R}$-matrix calculation by including all resonances up to $n=4$ for singly excited levels, while Whiteford et al. (2002) performed a 90-level intermediate-coupling frame transformation method (ICFT) $\boldsymbol{R}$-matrix calculation with radiative and auger damping for lithium-like iron up to the $n=3$ shell, including all doubly excited states with the exception of $1 s 3 l 3 l^{\prime}$ states.

\subsection{Beryllium Isoelectronic Sequence}

\subsubsection{III}

The $\mathrm{C}$ III CHIANTI model consists of all 20 levels from the six configurations $2 s^{2}, 2 s 2 p, 2 p^{2}$, and $2 s 3 l(l=s, p, d)$. Since version 1 of CHIANTI the radiative decay rates used for C III were from an unpublished calculation of one of the authors (P. R. Y.) using SUPERSTRUCTURE. These have now been replaced with the calculations of Tachiev \& Froese Fischer (1999). Eight transitions have $A$-values different from the previous calculation by more than $30 \%$. At $\log N_{e} / \mathrm{cm}^{-3}=10.0$ and $\log T / \mathrm{K}=4.8$, five of the levels show differences in level populations of $>10 \%$ on account of the new radiative data. The $2 s 2 p^{1} P_{1}$ level (which gives rise to the important resonance line at $977 \AA$ ) is reduced by $5.2 \%$.

$$
\text { 5.4.2. } \mathrm{O} \mathrm{v}
$$

The calculations of Kato et al. (1990) have been revisited by K. A. Berrington (2003, private communication), extending the calculation of effective collision strengths to a wider range of temperatures $(3.5 \leq \log T \leq 6.5)$, and to include the $2 p 3 l(l=$ $s, p, d)$ configurations. The model for $\mathrm{O} \vee$ thus now includes 46 levels of the $2 s^{2}, 2 s 2 p, 2 p^{2}, 2 s 3 l$, and $2 p 3 l(l=s, p, d)$ configurations. It was not possible to fit every transition accurately over the entire temperature range, and so the CHIANTI fits are valid over the restricted temperature range $4.2 \leq \log T \leq 6.3$. Only transitions involving the four lowest energy levels $\left(2 s^{2}{ }^{1} S_{0}\right.$ and $2 s 2 p^{3} P_{J}$ ) were fit, as these are the only transitions with a significant population for typical astrophysical conditions.

The $\mathrm{O} v$ radiative data have been revised with values from Tachiev \& Froese Fischer (1999) for the energetically lowest 20 levels (up to and including the $2 s 3 l$ levels). For 13 additional transitions, for which Tachiev \& Froese Fischer (1999) do not give data, we take values from Hibbert (1980). For all transitions involving the $2 p 3 l$ levels, radiative data are from Hibbert (1980). Previously, the CHIANTI radiative data were from an unpublished SUPERSTRUCTURE calculation (Dere et al. 1997). Comparisons with the new data show generally excellent agreement to 
better than $10 \%$, but for some of the $n=2$ to $n=3$ transitions there are differences between $10 \%$ and $90 \%$.

In terms of the strongest $\mathrm{O} v$ transitions, the new model results in the intercombination-to-resonance line ratio $(\lambda 1218.3 / \lambda 629.7)$ being within $5 \%$ of the previous model. The density-sensitive $\lambda 761.1 / \lambda 760.4$ ratio is up to $35 \%$ larger below a density of $10^{10} \mathrm{~cm}^{-3}$, but is within $3 \%$ above this density. The densitysensitive $\lambda 1371.3 / \lambda 1218.3$ ratio is between $10 \%$ and $20 \%$ larger than previously at all densities.

\subsection{3. $\mathrm{Ar} \mathrm{xV}$}

The observed energy of the $2 s 3 p^{3} P_{1}$ level has been added to the atomic model. Its value has been taken from the laboratory measurements of Lepson et al. (2003). All the rest of the data set for Ar XV is unchanged.

\subsubsection{Fe XXIII}

The atomic model for Fe XXIII has been greatly extended, to include data up to the $n=2 l 5 l^{\prime}$ complex $\left(l=s, p ; l^{\prime}=\right.$ $s, p, d, f, g)$, for a total of 166 fine-structure levels. Additional data for levels above the ionization limit belonging to the $1 s 2 s^{k} 2 p^{3-k}$ configuration $(k=0,1,2)$ allow us to include innershell transitions to the present CHIANTI model, as described in $\S 3.1$.

Observed energies (and wavelengths) for levels up to the $n=4$ complex are from the benchmark study of Del Zanna et al. (2005), where some new lines have been identified. Experimental energies for the $2 p 4 d^{1} P_{1}$ and $2 s 5 s{ }^{1} S_{0}$ are taken from the measurements in Landi \& Phillips (2005), while values for the $n=$ $2 l 5 l^{\prime}$ configurations come from Shirai et al. (2000).

Theoretical energies, wavelengths, and $A$-values for all levels with $n \leq 4$ have been obtained from a SUPERSTRUCTURE calculation that included empirical term energy corrections, described in Del Zanna et al. (2005). The $A$-values were calculated with the observed energies whenever available. The CHIANTI spline fits for transitions between levels up to $n \leq 4$ were obtained from the effective collision strengths calculated in the temperature range $2 \times 10^{6}-1.3 \times 10^{8} \mathrm{~K}$ by Chidichimo et al. (2005). Excitations from all the $n=2$ levels have been included, which is sufficient up to densities of $10^{20} \mathrm{~cm}^{-3}$. Chidichimo et al. (2005) performed an $\boldsymbol{R}$-matrix IRON Project calculation of all collision strengths for excitations to the $n=2,3,4$ levels. The data within the $n=2$ complex revise those previously published, while the $n=3,4$ represent the first $\boldsymbol{R}$-matrix calculations for this ion.

Theoretical energy levels, $A$-values, oscillator strengths, and collision strengths for the levels belonging to the $n=2 l 5 l^{\prime}$ configurations have been taken from the calculations of Landi \& $\mathrm{Gu}$ (2006a). Collision strengths have been calculated using the distorted-wave approximation for six values of the incident electron, from threshold to high energy, using FAC. $A$-values and oscillator strengths were calculated for all possible transitions from the $n=2 l 5 l^{\prime}$ levels.

\subsection{Boron Isoelectronic Sequence}

\subsubsection{II}

The only change in the CHIANTI data set for $\mathrm{C}_{\text {II }}$ is in the addition of $A$-values for seven transitions missing in the previous version of CHIANTI. The $A$-values were taken from version 2 of the NIST database (Fuhr et al. 1999). These transitions are $2 s 2 p^{2}{ }^{2} P_{J}-2 p^{3}{ }^{4} S_{3 / 2}, 2 p^{3}{ }^{4} S_{3 / 2}-2 s^{2} 3 s^{2} S_{1 / 2}$, and $2 s 2 p^{2}$ ${ }^{2} P_{J} 2 s^{2} 3 p^{2} P_{J}$.

$$
\text { 5.5.2. N III, O IV }
$$

The radiative data for these ions have been updated with the calculations of Tachiev \& Froese Fischer (2000) and Corrégé \& Hibbert (2002). The latter calculation provides data for all allowed transitions from the $2 s 2 p^{2}, 2 s^{2} 3 s$, and $2 s^{2} 3 d$ configurations to the ground $2 s^{2} 2 p$ configuration. The Tachiev \& Froese Fischer (2000) data are used for $2 s 2 p^{2}-2 p^{3}, 2 s 2 p^{2}-2 s^{2} 3 p, 2 s^{2} 3 s-$ $2 s^{2} 3 p$, and $2 s^{2} 3 p-2 s^{2} 3 d$ transitions. Further allowed transitions from the $2 s 2 p 3 s{ }^{4} P_{J}$ levels were also used for $\mathrm{O}$ IV only. The $A$-values for the ground forbidden transitions remain the same as in earlier versions of CHIANTI (Dere et al. 1997).

$$
\text { 5.5.3. Ne VI }
$$

The CHIANTI model for Ne vi has been completely revised and greatly extended in order to include the extensive data set developed by Mitnik et al. (2001). The atomic model now includes the 19 lowest configurations: $2 s^{2} 2 p, 2 s 2 p^{2}, 2 p^{3}, 2 s^{2} 3 l$, $2 s 2 p 3 l, 2 p^{2} 3 l, 2 s 2 p 4 l(l=s, p, d)$, and $2 s^{2} 4 l^{\prime}\left(l^{\prime}=s, p, d, f\right)$, for a total of 180 fine-structure levels. The experimental energies for the $n=2$ configurations come from Edlen (1983b), while those for all the other levels come from NIST, version 2 (Fuhr et al. 1999).

Theoretical energy values, oscillator strengths, $A$-values, and effective collision strengths are taken from Mitnik et al. (2001). The ICFT $\boldsymbol{R}$-matrix code was used on a large atomic model to provide Maxwellian-averaged collision strengths for all possible collisional transitions within the 180 levels in the model; data are provided in the $10^{4} \mathrm{~K} \leq T \leq 2 \times 10^{6} \mathrm{~K}$ temperature range, encompassing the range where $\mathrm{Ne}$ VI is expected to be abundant in plasmas under ionization equilibrium. Mitnik et al. (2001) quote good agreement with the Zhang et al. (1994) calculation previously used in CHIANTI, but the inclusion of many more levels allows us to take into account radiative cascading as a mechanism for level population.

\subsubsection{S XII, Ar XIV}

The energy levels for the $n=3$ configurations, originally from version 1 of the NIST database (Martin et al. 1995), have been updated for both ions. Observed energy values for S XII come from NIST version 3.0 (Ralchenko et al. 2005) and from the laboratory observations of Lepson et al. (2005). New energies for Ar XIV are taken from laboratory observations of Lepson et al. (2003) and Fawcett et al. (1971).

All other data for these two ions are unchanged.

$$
\text { 5.5.5. Fe XXIII }
$$

The Fe XXIII atomic model available in CHIANTI 4 has been extended to include all the configurations up to the $n=5$ complex, for a total of 39 configurations and 513 fine-structure levels. Thirty-five additional levels above the first ionization limit from the $1 s 2 s^{k} 2 p^{4-k}(k=0,1,2)$ have been included to take into account inner-shell transitions, as described in $\S 3.1$. Experimental energy levels come from a number of different sources that use both solar and laboratory measurements: Feldman et al. (2000), Sandlin et al. (1976), Brown et al. (2002), Landi \& Phillips (2005), and the NIST database, version 2 (Fuhr et al. 1999). In a few cases, the energies measured by Brown et al. (2002) and those available in NIST for the same level have been averaged together.

Radiative transition probabilities for all possible transitions in the adopted atomic model have been taken from the extensive calculations of Landi \& Gu (2006a), carried out using FAC. The collisional excitation rates for the lowest 204 levels from 
Badnell et al. (2001) have been retained, while collision strengths for transitions involving all the higher levels in the atomic model come from Landi \& Gu (2006a), who used FAC to calculate distorted-wave collision strengths at six values of the incident electron energy spanning from threshold to high energies. Landi \& Gu (2006a) also provide data for the lowest 204 levels, and for the $n=2,3$ complex they also include resonant excitation. A comparison between effective collision strengths from Badnell et al. (2001) and Landi \& Gu (2006a) yields an overall excellent agreement, with only a few exceptions.

\subsection{Carbon Isoelectronic Sequence}

5.6.1. O III

The CHIANTI model of O III consists of 46 levels of the $2 s^{2} 2 p^{2}, 2 s 2 p^{3}, 2 p^{4}$, and $2 s^{2} 2 p 3 l(l=s, p, d)$ configurations. The observed energy values for each of these levels have been replaced with the latest values from the NIST database (Ralchenko et al. 2005), and the wavelengths for all transitions have been recalculated. The new wavelengths give better agreement with the Bowen-fluoresced $n=3$ transitions of O III found in archival Hubble Space Telescope (HST) ultraviolet spectra of the symbiotic star RR Telescopii.

\subsection{2. $\mathrm{Ne} \mathrm{v}$}

The $A$-values of Bhatia \& Doschek (1993) for the ground $2 s^{2} 2 p^{2}$ configuration of $\mathrm{Ne} v$, introduced in version 1 of CHIANTI (Dere et al. 1997), have been replaced by the values of Galavís et al. (1997), except for the ${ }^{3} P_{1,2}-{ }^{1} D_{2}$ transitions, for which we retain the $A$-values of Storey \& Zeippen (2000). The ${ }^{1} D_{2}-{ }^{1} S_{0}$ $A$-value of Galavís et al. (1997) is a factor 2.5 greater than that of Bhatia \& Doschek (1993), leading to a $\lambda 1574 / \lambda 2974$ branching ratio of 0.376 (energy units). Due to the large wavelength separation of the lines, this ratio is valuable for determining the interstellar extinction toward astronomical objects (e.g., Penston et al. 1983).

\subsubsection{S XI}

The previous CHIANTI atomic model for S XI included data for the lowest 46 fine-structure levels; its main limitation was that collision strengths for levels belonging to the $2 s^{2} 2 p 3 l(l=s, p, d)$ were available only at one incident electron energy, so that the integration over the velocity distribution was rather uncertain. In the present version of the database, the CHIANTI model for $\mathrm{S}$ XI has been extended to 72 fine-structure levels in order to include three additional configurations: $2 s^{2} 2 p 4 l(l=s, p, d)$.

We have made use of the calculations performed by Landi \& Bhatia (2003a), who used the SUPERSTRUCTURE code to calculate energies, oscillator strengths, and $A$-values for all the levels and possible transitions within the adopted atomic model. Observed energy levels for the $n=2$ configurations have been taken from Edlen (1985), while the measured energies for several of the $n=3$ and $n=4$ configurations available in the literature were taken from the NIST database (ver. 3; Ralchenko et al. 2005), Bromage \& Fawcett (1977), and Lepson et al. (2005).

Landi \& Bhatia (2003a) also provide distorted-wave collision strengths calculated at five incident electron energy, thus allowing a much more accurate convolution of the collision strengths with the Maxwellian distribution of velocities. Landi \& Bhatia (2003a) demonstrated that resonances play a major role in the populations of levels in the ground configuration, so we replaced the Landi \& Bhatia (2003a) collision rates for the transitions among the six lowest levels with the $\boldsymbol{R}$-matrix rates obtained by Conlon et al. (1992). The other possible significant population mechanisms discussed by Landi \& Bhatia (2003a), photoexcitation, proton collisional excitation, and cascades, are already included in CHIANTI.

\subsection{4. $\mathrm{Cl}$ XII}

The three lowest configurations $\left(2 s^{2} 2 p^{2}, 2 s 2 p^{3}\right.$, and $\left.2 p^{4}\right)$ have been included in the CHIANTI model for $\mathrm{Cl}$ XII, for a total of 15 fine-structure levels. Experimental energy levels are taken from Edlen (1985). Zhang \& Sampson (1996) have provided a complete data set of theoretical energy levels, $A$-values, oscillator strengths, and collision strengths for these configurations, and their data are adopted here. Radiative data were provided only for allowed transitions, so forbidden transition probabilities for the ground configuration were taken from Nussbaumer \& Rusca (1979). Zhang \& Sampson (1996) provide relativistic distorted-wave collision strengths at six values of the scattered electron energy, from threshold to high energy, for all possible transitions.

\subsubsection{Fe XXI}

The Fe XXI atomic model available in CHIANTI 4 has been extended to include a large number of levels from the $n=4$ complex and the $2 s^{2} 2 p 5 l(l=s, p, d)$ configurations, for a total of 590 fine-structure levels, so that many observed lines could be predicted in the 8-11 $\AA$ wavelength range. Additional levels with energies above the ionization threshold from configurations $1 s 2 s^{k} 2 p^{5-k}(k=0,1,2)$ are also in the model, to include innershell transitions, as described in $\S 3.1$.

Experimental energies come from many different sources: Feldman et al. (2000), Brown et al. (2002), Fawcett et al. (1987), Landi \& Phillips (2005), and version 2 of the NIST database (Fuhr et al. 1999). The energies of a few levels are a combination of the NIST and Feldman et al. (2000) energies. Theoretical values come from FAC calculations from Landi \& Gu (2006a), who also calculated the $A$-values and oscillator strengths for all possible transitions used in the present version of CHIANTI.

Collisional excitation data are a combination of data already available in CHIANTI and new distorted-wave calculations from Landi \& Gu (2006a). Data for the $n=2$ and most of the $n=3$ complexes and for the $2 s^{2} 2 p 4 l(l=s, p, d, f)$ configurations are unchanged from CHIANTI 4, where the $\boldsymbol{R}$-matrix data from Badnell \& Griffin (2001) were used. Data for all other configurations come from the distorted-wave calculations carried out by Landi \& Gu (2006a) using FAC. No resonances were included for these high-energy configurations, and collision strengths were provided at six values of the incident electron energy, from threshold to high energy. Landi \& Gu (2006a) also provide data for all the configurations in the Badnell \& Griffin (2001) atomic model and include resonances for the $n=2$ and $n=3$ complexes; a comparison between the two data sets where both include resonances is discussed by Landi \& Gu (2006a), who find an overall very good agreement, with a few exceptions that have no influence on level populations and line emissivities.

\subsection{Nitrogen Isoelectronic Sequence}

5.7.1. $\mathrm{Mg}$ vI

Earlier versions of the CHIANTI database already included data for $\mathrm{Mg}$ VI that allowed the calculation of line emissivities for the 22 fine-structure levels of the lowest four configurations. Now the Mg vi atomic model has been extended to six configurations, for a total of 72 fine-structure levels. The configurations included are now $2 s^{2} 2 p^{3}, 2 s 2 p^{4}, 2 p^{5}$, and $2 s^{2} 2 p^{2} 3 l(l=s, p, d)$. Observed energy levels for the $n=2$ complex are taken from 
Edlen (1984), and those for the $n=3$ levels come from the NIST database (Fuhr et al. 1999). Theoretical energy levels, $A$-values, and weighted oscillator strengths come from the 24-configuration model by Landi \& Bhatia (2006a). These replace the data from Zhang \& Sampson (1999) and Merkelis et al. $(1997,1999)$ in the previous CHIANTI version, as Landi \& Bhatia (2006a) demonstrated that the Zhang \& Sampson (1999) $A$-values were slightly overestimated relative to more sophisticated calculations, while the latter were in good agreement with their results.

Collision rates for the lowest four configurations are taken from Ramsbottom \& Bell (1997) and consist of $\boldsymbol{R}$-matrix calculations of Maxwellian-averaged collision strengths calculated in the $5.0 \leq \log T_{e} \leq 6.1$ temperature range $(T$ in $\mathrm{K})$. These data replace the distorted-wave calculations from Bhatia \& Young (1998). Data for the remaining two configurations $\left(2 s^{2} 2 p^{2} 3 l\right.$, $l=p, d)$ are taken from Landi \& Bhatia (2006a): these data consist of distorted-wave collision strengths calculated for five values of the incident electron energy. Oscillator strengths for these two configurations come from the same six-configuration calculation that produced the collision strengths, for consistency.

The inclusion of the 50 additional levels in these two configurations alters the emissivity of the lines emitted by the $2 s^{2} 2 p^{2} 3 s$ configuration due to radiative cascades to those levels; also, resonances from the Ramsbottom \& Bell (1997) calculation enhance the population of these levels. No changes were found for the lines in the $n=2$ complex.

\subsection{2. $\mathrm{S} x$}

The $\mathrm{S} x$ atomic model already available in CHIANTI has been extended in order to include all the levels in the $2 s^{2} 2 p^{2} 3 l(l=$ $p, d)$, not available in the previous version of the database. The total number of fine-structure levels has been brought to 72 from the 22 in the previous versions. Observed energy levels for the $n=2$ complex are taken from Edlen (1984), while a complete set of energies for the $2 s^{2} 2 p^{2} 3 l(l=s, p, d)$ configurations has been taken from the laboratory measurements of Kink \& Engström (1999). Theoretical energy levels come from the 24-configuration calculation of Bhatia \& Landi (2003a).

Bhatia \& Landi (2003a) provide a complete set of energy levels, $A$-values, and oscillator strengths for all transitions among the 72 fine-structure levels, calculated using a 24-configuration atomic model. These data are adopted in the present version of CHIANTI. They also provide a complete set of distorted-wave collision strengths for all possible transitions, calculated at five values of the incident electron energy. However, for the four lowest configurations ( $n=2$ complex and the $2 s^{2} 2 p^{2} 3 s$ configuration), we have replaced Bhatia \& Landi (2003a) data with the $\boldsymbol{R}$-matrix calculations from Bell \& Ramsbottom (2000). Bhatia \& Landi (2003a) show that resonances are very important for the calculation of $\mathrm{S} x$ level populations.

Proton rates for all the transitions between the levels in the ground configuration of $\mathrm{S} x$ have been reported by Bhatia \& Landi (2003a), using a semiclassical Coulomb excitation, as described by Kastner \& Bhatia (1979). Bhatia \& Landi (2003a) show that proton rates are an important factor to be considered when calculating level populations, altering results by up to $40 \%$. These rates are now included in CHIANTI.

\subsection{3. $\mathrm{Cl} \mathrm{XI}$}

The atomic model of $\mathrm{Cl} \mathrm{XI}$, a new entry for CHIANTI, includes the lowest three configurations $\left(2 s^{2} 2 p^{3}, 2 s 2 p^{4}\right.$, and $\left.2 p^{5}\right)$, for a total of 15 fine-structure levels. The experimental energy levels are taken from Edlen (1984), while their theoretical levels come from Merkelis et al. (1999) and Merkelis et al. (1997) for the ground configuration and for the two excited configurations, respectively. These authors also provide the oscillator strengths and $A$-values adopted here. Collisional data come from Zhang \& Sampson (1999), who provide relativistic collision strengths at six values of the scattered electron energy from threshold to high energy. Oscillator strengths from Zhang \& Sampson (1999) have been used to scale the collisional data, for consistency.

$$
\text { 5.7.4. Ar XII }
$$

All the data for Ar XII available in the older version of the CHIANTI database have been replaced with the extensive calculations done by Eissner et al. (2005). The configurations and levels adopted in the present version are unchanged and include the 72 lowest fine-structure levels from the configurations $2 s^{2} 2 p^{3}, 2 s 2 p^{4}, 2 p^{5}$, and $2 s^{2} 2 p^{2} 3 l(l=s, p, d)$. The observed energy levels come from Edlen $(1984 ; n=2)$ and the NIST database, version 2 (Fuhr et al. 1999; $n=3$ ). Theoretical energy levels, as well as oscillator strengths and $A$-values for all possible transitions between the levels in the model, have been taken from the 24-configuration SUPERSTRUCTURE calculation by Eissner et al. (2005).

Collisional data for the $n=3$ levels in the previous version of the database included distorted-wave collision strengths calculated at one single energy, so the calculation of the collision rate was uncertain. The present version replaces these data with the distorted-wave calculations from Eissner et al. (2005), which are also used for the transitions among the $n=2$ levels. Eissner et al. (2005) provide collision strengths at five incident energies and allow an accurate calculation of the convolution of the collision strengths with the Maxwellian distribution of velocities.

\subsubsection{Ca XIV}

The distorted-wave collision strengths from the previous version of the CHIANTI database have been replaced with new distorted-wave calculations from Landi \& Bhatia (2005b), who extended the atomic model by adding four new configurations to the $n=2$ already available: $2 s^{2} 2 p^{2} 3 l(l=s, p, d)$ and $2 s 2 p^{4} 3 s$. Data for these additional configurations were previously unavailable in the literature and allow the prediction of several lines in the UV and X-ray wavelength range that can be used for plasma diagnostics.

Observed energy levels for the first six levels come from Feldman et al. (2000), while those for the remaining $n=2$ levels are taken from Edlen (1984). Observed energy levels for the $n=3$ configurations are from Fuhr et al. (1999) and Kaufman et al. (1982). Theoretical values are taken from the 24-configuration calculation of Landi \& Bhatia (2005b), who also provide the oscillator strengths and $A$-values for all the possible transitions within the adopted atomic model used here.

Landi \& Bhatia (2005b) calculated distorted-wave collision strengths at five values of the incident electron energy that replace the values from Zhang \& Sampson (1999) used in the previous version 4 . A comparison between the two computations shows agreement within $30 \%$, except for the two levels in the $2 p^{5}$ configuration, where Zhang \& Sampson (1999) seem to underestimate the collision strengths.

$$
\text { 5.7.6. Fe } \mathrm{xx}
$$

The Fe xx atomic model available in CHIANTI 4 has been expanded to include 27 configurations for a total of 725 finestructure levels. All the configurations belonging to the $n=2$, $n=3$, and $n=4$ complex have been included, together with the $2 s^{2} 2 p^{2} 5 l(l=s, p, d)$ configurations. Sixteen additional levels with energies higher than the ionization limit have been added 
from the $1 s 2 s^{k} 2 p^{6-k}$ configurations $(k=0,1,2)$ to take into account inner-shell transitions, as described in $\S 3.1$. Experimental energy values have been taken from several different sources that used both solar and laboratory observations: Kucera et al. (2000), Edlen (1984), Landi \& Phillips (2005), and Shirai et al. (2000). They are available for many levels in the $n=3$ complex, and for a few more $n=4$ and $n=5$ levels. Theoretical energies come from the calculations of Landi \& Gu (2006a).

Landi \& Gu (2006a) provided a complete data set of oscillator strengths, $A$-values, and collision rates for all the levels in the atomic model below the ionization limit; these data are used in CHIANTI 5. Collisional excitation rates were calculated in the distorted-wave approximation for six values of the incident electron energy between threshold and high energies, but resonances for transitions in the $n=2$ and $n=3$ complex, calculated by $\mathrm{Gu}$ (2003) using the independent-process, isolated-resonance approximation, were included in the total collision rates. However, for the ground configuration the $\boldsymbol{R}$-matrix effective collision strengths from McLaughlin \& Kirby (2001) have been used: they agree with the Landi \& Gu (2006a) data within $20 \%$ in the temperature range where the latter are calculated, but they are provided by the authors at temperatures lower than those considered by Landi \& Gu (2006a), so they allow a better calculation of the collision rates at the very low temperature limit. $\boldsymbol{R}$-matrix effective collision strengths from Butler \& Zeippen (2001a) were used in place of the Landi \& $\mathrm{Gu}$ (2006a) data for the $2 p^{6} 2 P$ doublet, for which Landi \& Gu (2006a) do not include some important resonances. Landi \& Gu (2006a) data replace the effective collision strengths from Butler \& Zeippen (2001a) available in CHIANTI 4. A comparison between the Landi \& Gu (2006a) data and the Butler \& Zeippen (2001a) data set showed an overall good agreement, but some problems were found in the background collision strengths of the latter for a few transitions giving rise to observed lines, which caused the total collision rate to be overestimated.

\subsection{Oxygen Isoelectronic Sequence}

\subsubsection{Ne III}

Landi \& Bhatia (2005a) noted that the calculations by Bhatia et al. (2003b) used in version 4 of the database were carried out with a limited atomic model that omitted the $2 s^{2} 2 p^{2} 3 p$ configuration. This had two effects on the data: the wave functions for the $2 s^{2} 2 p^{2} 3 l(l=s, d)$ levels were of limited accuracy, and several transitions to and from the $2 s^{2} 2 p^{2} 3 p$ configuration were missing.

The atomic model of $\mathrm{Ne}$ III has now been extended to include the levels of the $2 s^{2} 2 p^{2} 3 p$ as well as the $2 p^{6}$ configurations. The total number of fine-structure levels is now 86 ; their experimental energies have been taken from version 2 of the NIST database (Fuhr et al. 1999). All the theoretical energies and transition rates come from the extensive calculations performed by Landi \& Bhatia (2005a). Theoretical energies, oscillator strengths, and $A$-values are calculated with a 24-configuration atomic model that greatly improves the results over the Bhatia et al. (2003b) calculation. The $A$-values for the ${ }^{3} P_{1}-{ }^{1} S_{0}$ and ${ }^{1} D_{2}-{ }^{1} S_{0}$ are taken from the laboratory measurements of Daw et al. (2000).

Resonances are an important contributor to electron collisional excitation in Ne III, so we have used the close coupling calculations of McLaughlin \& Bell (2000) for the 10 transitions within the ground configuration. Collision strengths for all other transitions are provided at five incident electron energies and are calculated with a six-configuration model; their values represent an improvement over the previous calculation, as noted by Landi \& Bhatia (2005a).
5.8.2. $\mathrm{Mg} \mathrm{v}$

The data set for $\mathrm{Mg} \mathrm{v}$ previously available in CHIANTI, mostly composed of unpublished calculations by A. K. Bhatia, has been updated to include the extensive calculations of Bhatia et al. (2006). The atomic model has now been extended to 86 fine-structure levels from the $2 s^{2} 2 p^{4}, 2 s 2 p^{5}, 2 p^{6}$, and $2 s^{2} 2 p^{3} 3 l$ $(l=s, p, d)$. Observed energy levels for the $n=2$ configurations have been taken from Edlen (1983a), while the values for the $2 s^{2} 2 p^{3} 3 l$ configurations come from the NIST database, version 2 (Fuhr et al. 1999). Theoretical energy levels, oscillator strengths, and $A$-values come from Bhatia et al. (2006); transition rates have been provided for all possible transitions between the 86 levels.

Collision strengths also come from Bhatia et al. (2006), and they have been calculated at five incident electron energies using the distorted-wave approximation. However, Butler \& Zeippen (1994) calculated Maxwellian-averaged collision strengths for all the transitions within the ground configuration using the $\boldsymbol{R}$-matrix method; they show that resonances play an important role in collisional excitation of the lowest five levels. However, Butler \& Zeippen (1994) provided data for temperatures lower than $10^{5} \mathrm{~K}$, while the $\mathrm{Mg} \mathrm{v}$ maximum abundance temperature is $2.5 \times 10^{5} \mathrm{~K}$ under conditions of equilibrium (Mazzotta et al. 1998). In order to extend the range of validity of the Butler \& Zeippen (1994) data set, we have merged their data with effective collision strengths at $T>10^{5} \mathrm{~K}$ obtained integrating the Bhatia et al. (2006) collision strengths, and inserted the resulting effective collision strengths in CHIANTI; the two data sets merge without difficulties, and no inconsistencies were found for any transition.

$$
\text { 5.8.3. Al VI }
$$

Level energies and radiative data have been updated from the last version of the CHIANTI database, without changing the size of the 10-level atomic model, which includes the three $n=2$ configurations $2 s^{2} 2 p^{4}, 2 s 2 p^{5}$, and $2 p^{6}$. Experimental energies now come from Edlen (1983a), while theoretical values, as well as oscillator strengths and $A$-values, are taken from the SUPERSTRUCTURE calculations of Landi (2005). These replace the interpolated values available in the previous version of CHIANTI.

\subsubsection{Si VII}

The Si VII atomic model in the present version has been extended to include the $2 s^{2} 2 p^{3} 3 l(l=s, p, d)$ configurations, previously unavailable in the literature. Also, the data for the 10 levels within the $n=2$ complex have been renewed from version 4 . The new Si vII model includes 86 fine-structure levels. Experimental energy values are available for nearly all the levels in the atomic model, and they come from the NIST database, version 2 (Fuhr et al. 1999), and from the laboratory or solar measurements of Kink et al. (1997) and Kink \& Engström (1997). The theoretical energy levels are provided by Bhatia \& Landi (2003b) for all levels.

Oscillator strengths for the transitions within the ground configuration come from Tachiev \& Froese Fischer (2002) and Froese Fischer \& Saha (1983); the values for all the other transitions have been taken from Bhatia \& Landi (2003b). These authors were the first to provide transition rates and collision strengths for the $2 s^{2} 2 p^{3} 3 l$ configurations, and their values are adopted here for collisional excitation of all the levels in Si vII. The distortedwave collision strengths calculated by Bhatia \& Landi (2003b) at five incident electron energies also replace the older values of 
Bhatia et al. (1979) for the $n=2$ levels previously available in CHIANTI.

$$
\text { 5.8.5. P VIII, K XII }
$$

The data sets available in the previous versions have been updated with new calculations for the $A$-values and oscillator strengths, and for the collision rates. The radiative data for the ground configuration have been taken from Tachiev \& Froese Fischer (2002) and Froese Fischer \& Saha (1983), while data from Landi (2005) for the excited configuration replace the older calculations by Fawcett (1986).

Relativistic distorted-wave collision strengths for all transitions within the $n=2$ configurations were calculated by Zhang $\&$ Sampson (2002) at six values of the scattered electron energy, encompassing the entire range from threshold to high energy. These data replace the interpolated effective collision strengths in the previous version of the database. In the case of P VIII, they also replace the $\boldsymbol{R}$-matrix calculations of Butler \& Zeippen (1994). Oscillator strengths used in the scaling of the effective collision strengths also are taken from Zhang \& Sampson (2002), for consistency. Although Butler \& Zeippen (1994) data include resonances, neglected by Zhang \& Sampson (2002), they have been replaced in CHIANTI since they were provided only for temperatures lower than $10^{5} \mathrm{~K}$, much below the temperature range for which $\mathrm{P}$ VIII has nonnegligible abundance, so their extrapolation to these higher temperatures has been estimated to be less reliable than the distorted-wave data.

\subsubsection{S IX}

The energy levels, transition rates, and $A$-values for $\mathrm{S}$ Ix are the same as in the previous version of the CHIANTI database, with only a few exceptions. The experimental energies have been replaced with values from Edlen (1983a) for the $n=2$ levels, and from Jupén \& Engström (1997) and Lepson et al. (2005) for the $n=3$ levels. Radiative data for the ground configuration from Bhatia \& Landi (2003c) have been replaced with values from Tachiev \& Froese Fischer (2002) and Froese Fischer \& Saha (1983). All the rest of the data are unchanged.

\subsection{7. $\mathrm{Cl}$ v, Ti xv, Cr xvII, Mn xvIII, Co xx, Zn xxII}

The atomic model for these ions still includes the $n=2$ configurations, for a total of 10 fine-structure levels. Experimental energy levels have been taken from Edlen (1983a), and theoretical values come from Landi (2005). Oscillator strengths and $A$-values are also taken from Landi (2005), while collision strengths are from the relativistic distorted-wave calculations of Zhang \& Sampson (2002). These authors provided collision strengths calculated at six values of the scattered electron energy, from close to threshold to high energies, thus allowing a very accurate convolution with the Maxwellian distribution of electron velocities. Oscillator strengths used in the scaling of the effective collision strengths are also taken from Zhang \& Sampson (2002), for consistency. These data replace either data interpolated along the isoelectronic sequence or older data sets. $\mathrm{Cl} \mathrm{v}$, Co Xx, and $\mathrm{Zn}$ XXIII are new entries in the CHIANTI database.

$$
\text { 5.8.8. Ar XI }
$$

The Ar XI atomic model in CHIANTI has been extended to include the three configurations $2 s^{2} 2 p^{3} 3 l(l=s, p, d)$; the data for the configurations already included in CHIANTI have also been updated. The atomic model now includes the six lowest configurations $\left(2 s^{2} 2 p^{4}, 2 s 2 p^{5}, 2 p^{6}\right.$, and $\left.2 s^{2} 2 p^{3} 3 l\right)$ for a total of 86 fine-structure levels. Experimental levels for the $n=2$ levels come from Edlen (1983a), while the values for the $n=3$ levels have been taken from Curdt et al. (2004), Kelly (1987), Lepson et al. (2005), and Fawcett et al. (1971). Theoretical energy levels, as well as oscillator strengths, $A$-values, and collision strengths, have been taken from the calculations of Landi \& Bhatia (2006b).

Landi \& Bhatia (2006b) provide oscillator strengths, $A$-values, and collision strengths for all transitions within the 86 levels in the atomic model, and their values replace the older calculations from Bhatia et al. (1979) previously used. Landi \& Bhatia (2006b) used a 24-configuration atomic model to calculate radiative transition rates that greatly improves their accuracy. Collision strengths have been calculated using the distortedwave approximation with a six-configuration atomic model, for five values of the incident electron energy. Butler \& Zeippen (1994) carried out close coupling calculations of collision rates for transitions in the ground configuration, but they provide values only up to $T=10^{5} \mathrm{~K}$, much lower than the temperature of maximum abundance of $\operatorname{Ar}$ XI $\left(\simeq 2 \times 10^{6} \mathrm{~K}\right)$, so these data have not been used here.

\subsubsection{Ca XIII}

The unpublished calculations of A. K. Bhatia used in the previous version of CHIANTI have been replaced by the extensive calculations by Landi \& Bhatia (2006c). The 10-level atomic model with the $n=2$ configurations has been extended to include the $2 s^{2} 2 p^{3} 3 l(l=s, p, d)$ configuration for a total of 86 fine-structure levels, so that lines in the UV and X-ray range originated by these configurations could now be predicted. The experimental energy levels have been taken from several sources: Edlen (1983a) for the $n=2$ levels, with the exceptions of levels ${ }^{1} D_{2}$ and ${ }^{1} S_{0}$ in the ground configuration, whose energies were measured by Feldman et al. (2000); energies for the $n=3$ levels come from Acton et al. (1985), Doschek et al. (1973), and Fawcett \& Hayes (1975).

Theoretical energy levels, oscillator strengths, $A$-values, and collision strengths were provided by Landi \& Bhatia (2006c). Transition rates were available for all possible transitions within the 86 levels in the model; collision strengths were calculated at five values of the incident electron energies under the distortedwave approximation. Comparison with the $L S \boldsymbol{R}$-matrix collision rates of Baliyan \& Bhatia (1994) yielded good agreement, but it is likely that resonances are important contributors to the population of the $n=2$ levels, and their neglect in the Landi \& Bhatia (2006c) model is the main limitation of the present data set, as discussed in Landi \& Bhatia (2006c).

\subsubsection{Fe XIX}

The CHIANTI 4 model for Fe XIX has been expanded to include many more configurations, so the new model includes all the configurations in the $n=2, n=3$, and $n=4$ complexes and the additional configurations $2 s^{2} 2 p^{3} 5 l(l=s, p, d)$, for a total of 630 fine-structure levels. Two additional configurations above the ionization threshold $\left(1 s 2 s^{2} 2 p^{5}\right.$ and $\left.1 s 2 s 2 p^{6}\right)$ have been included to predict emissivities for inner-shell transitions ( $\S 3.1)$. Observed energy levels are taken from Shirai et al. $(2000 ; n=2)$, and from Landi \& Phillips (2005) and Brown et al. (2002) for the other configurations.

Theoretical energy levels, oscillator strengths, $A$-values, and collision rates are taken from the calculations of Landi \& Gu (2006a), with the only exception of the effective collision strengths for the transitions to the $2 p^{6}{ }^{1} S_{0}$, which come from Butler \& Zeippen (2001b). Landi \& Gu (2006a) calculated collision rates with the distorted-wave approximation for six values of the incident electron energy from threshold to high energy; effective collision strengths obtained by convolving the results with 
a Maxwellian distribution of velocities were added to the resonant excitation rates calculated by $\mathrm{Gu}(2003)$ for the $n=2$ and $n=2$ complexes, and the total rates were included in the present version of CHIANTI.

A comparison between the Butler \& Zeippen (2001b) and Landi $\& \mathrm{Gu}$ (2006a) data sets shows very good agreement in most cases, but also a few disagreements affecting some observed lines. A detailed investigation of the original collision strengths from Butler $\&$ Zeippen (2001b) has revealed some problems in the convergence of the partial-wave summation in some transitions, so their final collision rates were underestimated.

\subsubsection{Ni XXI}

The atomic model of Ni XXI adopted in the previous version of CHIANTI is the same as the Ca XIII one described in the previous section, except that it does not include the $2 s^{2} 2 p^{3} 3 p$ configuration (Bhatia et al. 2003a). This configuration was left out of the atomic model both in the atomic structure and in the electron-ion collision problems. However, this omission might affect significantly the resulting transition rates and the collision strengths. Improved calculations that include this configuration are under way, but they are currently unavailable to us. In order to improve at least the $A$-values and oscillator strengths, we have carried out a SUPERSTRUCTURE calculation including 24 configurations, using the same atomic model adopted for the new data of the rest of the O-like sequence. The configurations included are $2 s^{2} 2 p^{4}, 2 s 2 p^{5}, 2 p^{6}, 2 s^{2} 2 p^{3} 3 l, 2 s 2 p^{4} 3 l, 2 p^{5} 3 l, 2 s^{2} 2 p^{3} 4 l^{\prime}$, $2 s 2 p^{4} 4 l^{\prime}$, and $2 p^{5} 4 l^{\prime}$, with $l=s, p, d$ and $l^{\prime}=s, p, d, f$. $A$-values have been corrected to account for experimental wavelengths whenever these were available. Theoretical energy levels, oscillator strengths, and $A$-values from this calculation replace those in the previous version of the database. $A$-values from the $2 s^{2} 2 p^{3} 3 s$ and $2 s^{2} 2 p^{3} 3 d$ are sometimes different from the values published by Bhatia et al. (2003a). In the .SPLUPS file, the original oscillator strengths from Bhatia et al. (2003a) have been retained, for consistency with the collisional calculation and the scaling.

\subsection{Fluorine Isoelectronic Sequence}

\subsubsection{Fe XVIII}

Fe XVIII has been greatly expanded in the present version of CHIANTI by including the $n=4$ complex and the $2 s^{2} 2 p^{4} 5 l$ $(l=s, p, d)$ configurations, thus bringing the total number of finestructure levels to 336 . The $1 s 2 s^{2} 2 p^{6}$ configuration, whose energy is higher than the $\mathrm{Fe}$ xvIII ionization limit, is also included to take into account a few inner-shell transitions (see $\S 3.1$ ). The observed energy levels for the $n=2$ configurations come from the line lists of Shirai et al. (2000), while energies for all the other excited levels are taken from Landi \& Phillips (2005).

Landi \& Gu (2006a) provide a complete data set of theoretical energy levels, $A$-values, oscillator strengths, and collision rates for all the levels in the present atomic model. Collision rates are obtained by summing the distorted-wave collision strengths calculated by Landi \& $\mathrm{Gu}(2006 \mathrm{a})$ at six values of the incident electron energy from threshold to high energies, with the resonant excitation collision rates by $\mathrm{Gu}$ (2003) for the $n=3$ complex. $\mathrm{Gu}$ (2003) has been the first to include resonant excitation for the $n=3$ data in Fe XVIII, and a comparison with the emissivities predicted with the previous version of CHIANTI, which used relativistic distorted-wave data with no resonances from Sampson et al. (1991), shows significant changes in the level populations and predicted line emissivities. The effective collision strengths for the transition in the ground ${ }^{2} P_{J}$ multiplet from Berrington et al. (1998) has been retained; the comparison with the Landi \& $\mathrm{Gu}$ (2006a) results shows agreement within 35\%.

\subsection{Neon Isoelectronic Sequence}

\subsubsection{Ar IX}

The observed energy of the $2 s 2 p^{6} 3 p^{1} P_{1}$ level has been added to the atomic model. Its value has been taken from the laboratory measurements of Lepson et al. (2003). All the rest of the data set for Ar IX is unchanged.

\subsubsection{Fe XVII}

The new CHIANTI model for Fe XVII has a few new features that improve it from version 4 . The total number offine-structure levels has been brought to 267 by including the $n=5$ and $n=6$ complexes and the $2 s^{2} 2 p^{5} 7 l(l=s, p, d)$ configurations, thus allowing CHIANTI to predict a number of observed transitions previously unavailable. The observed energies and all the transition rates have been changed, in order to include new observed values and the new data set from Landi \& Gu (2006a).

Observed energies come from several sources: Shirai et al. (2000), Feldman et al. (1998), Thomas \& Neupert (1994), Young et al. (1998), Brosius et al. (1998), and Landi \& Phillips (2005). Theoretical energies, $A$-values, oscillator strengths, and collisional rates are taken from Landi \& Gu (2006a). Collision rates from the ground to all excited levels were calculated using the distorted-wave approximation, with the exception of the $n=3$ levels, for which the resonant excitation rates from $\mathrm{Gu}$ (2003) were added to the direct excitation rates. Collision strengths were calculated for six values of the incident electron energy, from threshold to high energy. The presence of resonances, along with recombination into excited levels (see $\S 2.3$ ), has important consequences for the level populations, as shown by Doron $\&$ Behar (2002) and Landi \& Gu (2006a), and helps in solving a few discrepancies between observed and predicted spectra.

Recently, Brown et al. (2005) have measured the collision rate for the allowed transition $2 p^{6}{ }^{1} S_{0}-2 s^{2} 2 p^{5} 3 d^{1} P_{1}$, giving rise to the very strong line observed at $15.015 \AA$, and found it to be significantly lower than all calculations available in the literature, including the present one (by $\simeq 30 \%-50 \%$ ). This lower excitation rate would help bring the predicted intensity of this line in agreement with observations. However, measurements of this rate were done at only one excitation energy, so that it was not possible to determine its temperature dependence; in the present version of CHIANTI we preferred not to include these collision rates, and wait for more complete measurements.

\subsection{Magnesium Isoelectronic Sequence}

\subsubsection{Al II}

Al II is a new addition to CHIANTI, and the model features 20 levels of the $3 s^{2}, 3 s 3 p, 3 p^{2}, 3 s 3 d, 3 s 4 s$, and $3 s 4 p$ configurations. Effective collision strengths are from Aggarwal \& Keenan $(1994 b, 1998)$ and are tabulated at 16 temperatures from 5000 to $100,000 \mathrm{~K}$. The $L S$ collision strengths from Aggarwal \& Keenan (1994b) have, where necessary, been split according to their statistical weights to provide fine-structure collision strengths. The oscillator strengths that are required to derive the hightemperature limit points necessary for the CHIANTI fits are from Aggarwal \& Keenan (1994a) for seven allowed transitions, and from radiative data derived using SUPERSTRUCTURE (see below) for all other allowed transitions. Only those transitions involving the five $3 s^{2}{ }^{1} S_{0}, 3 s 3 p{ }^{3} P_{J}$, and $3 p^{2}{ }^{1} D_{2}$ levels were fit for CHIANTI, as these are the only levels with significant population in astrophysical conditions. 
$A$-values for the $3 s^{2}{ }^{1} S_{0}-3 s 3 p{ }^{1} P_{1}, 3 s 3 p{ }^{3} P_{1}-{ }^{3} P_{2}$, and $3 s^{2}{ }^{1} S_{0}-$ $3 s 3 p^{3} P_{2}$ transitions have been taken from Zou \& Froese Fischer (2001). The lifetime of the $3 s 3 p{ }^{3} P_{1}$ level has been measured experimentally by Träbert et al. (1999b), and the derived intercombination transition $\left(3 s^{2}{ }^{1} S_{0}-3 s 3 p^{3} P_{1}\right) A$-value is used in CHIANTI. All other radiative data have been obtained from a SUPERSTRUCTURE calculation performed by one of the authors (P. R. Young). This calculation included 50 configurations up to $n=5$. $L S$ oscillator strengths were compared with those of Tayal \& Hibbert (1984), and good agreement was found.

Experimental energy levels are available for all levels and were obtained from the NIST database.

\subsubsection{Fe xv}

The atomic model for Fe $\mathrm{xv}$ has been expanded to include 155 fine-structure levels and to include new, accurate transition rates published in the literature since the last CHIANTI release. The new configurations included in the present atomic model are the $3 p 4 l(l=p, d, f), 3 s 5 l$, and $3 p 5 l(l=s, p, d, f, g)$, which complement those already available in CHIANTI. Observed energy levels are taken from several different sources: Shirai et al. (2000), Eissner et al. (1999), Reader \& Sugar (1975), and version 3 of NIST (Ralchenko et al. 2005). Theoretical energy levels, oscillator strengths, and $A$-values are taken from the calculations of Landi \& $\mathrm{Gu}(2006 \mathrm{~b})$, who also provide collision excitation rates among all levels in the atomic model.

Collision strengths calculations have been carried out by Landi $\& \mathrm{Gu}(2006 \mathrm{~b})$ in the distorted-wave approximation, but for the $3 s^{2}-3 s 3 p$ and $3 s^{2}-3 p^{2}$ transitions resonant excitation was taken into account using the independent-process, isolated-resonance approximation. However, for the lowest 45 fine-structure levels we have made use of the results from Berrington et al. (2005), who made use of a fully relativistic $\boldsymbol{R}$-matrix code (DARC) and a semirelativistic Breit-Pauli $\boldsymbol{R}$-matrix code to calculate collision strengths as a function of energy with unprecedented energy resolution to properly resolve resonances.

\subsection{Aluminum Isoelectronic Sequence}

\subsubsection{S IV}

New calculations of radiative decay rates for allowed transitions between the ground $3 s^{2} 3 p^{2} P_{J}$ levels and the 11 levels of the $3 s 3 p^{2}, 3 s^{2} 3 d$, and $3 s^{2} 4 s$ configurations have been presented by Hibbert et al. (2002). Significant differences were found for the $3 s^{2} 3 p^{4} P_{J^{-}}-3 s 3 p^{2}{ }^{4} P_{J^{\prime}}$ intercombination transitions over the values of Tayal (1999) used in earlier versions of CHIANTI. For all other transitions, however, good agreement with Tayal (1999) was found, except for transitions with small oscillator strengths. An error in Table 8 of Hibbert et al. (2002) has been corrected: the $A$-value for the $3 s^{2} 3 p^{2} P_{3 / 2}-3 s 3 p^{2} D_{3 / 2}$ was incorrectly listed as $2.251 \times 10^{8} \mathrm{~s}^{-1}$ instead of $2.251 \times 10^{7} \mathrm{~s}^{-1}$.

$$
\text { 5.12.2. Ca VIII }
$$

Data for Ca vIII were nearly absent from the literature, with the only exception of the transition within the doublet of the ground configuration, and the $A$-values for a few transitions, so this ion was not part of earlier versions of CHIANTI despite its potential for element abundance diagnostics.

Recently, Landi et al. (2004) calculated a complete set of atomic data and transition rates for $\mathrm{Ca}$ VIII, and these data are included in the present version of CHIANTI. Landi et al. (2004) present data for level energies, transition rates, and Maxwellianaveraged collision strengths for all levels in the five lowest configurations of $\mathrm{Ca}$ vIII, for a total of 40 fine-structure levels.
Observed energy levels are taken from Churilov \& Levashov (1993), and these data have been used to calculate the $A$-values from the line strengths. Among the possible transitions within the 40 energy levels in the model, only the $A$-values higher than $0.01 \%$ of the total transition probability of the upper state of that transition were used. The scattering calculation has been carried out using the $\boldsymbol{R}$-matrix method for incident electron energies up to $80 \mathrm{ryd}$, and a fine grid of 9000 points in the resonant energy range to properly resolve resonances. Landi et al. (2004) demonstrate that resonances are very important at low temperatures.

\subsection{Silicon Isoelectronic Sequence}

$$
\text { 5.13.1. Ca VII }
$$

Ca vII lines have been observed by a number of authors in solar quiet and active region, but no complete data set was available in the literature until recently, so this ion was not included in previous versions of CHIANTI, despite its great potential for element abundance diagnostics.

Landi \& Bhatia (2003b) published a complete data set of atomic data and transition rates for the lowest three configurations of $\mathrm{Ca}$ VII, for a total of 27 fine-structure levels. These data are adopted in version 5. Calculations are carried out using the University College London suite of codes.

Landi \& Bhatia (2003b) adopt a 25-configuration atomic model to calculate energies and wave functions, and radiative transition probabilities. The latter are corrected using the observed energies where these are available, taken from the NIST database (Fuhr et al. 1999) and Kelly (1987). $A$-values are provided for all possible transitions between the 27 levels.

Collisional calculations have been carried out adopting a more limited atomic model because of computer memory limitations: only the five lowest energy configurations have been included $\left(3 s^{2} 3 p^{2}, 3 s 3 p^{3}, 3 s^{2} 3 p 3 d, 3 p^{4}\right.$, and $\left.3 s^{2} 3 d^{2}\right)$. Collision strengths were calculated in the distorted-wave approximation at seven incident electron energies: 8, 10, 15, 20, 30, 40, and 60 ryd.

Landi \& Bhatia (2003b) point out that the main limitations of their collisional calculations are the neglect of resonances and of high-energy configurations. The effects of resonances are impossible to assess, due to the lack of collision rates that include them in the literature; the effects of the latter depend on the importance of these configurations for the wave functions of the 27 levels considered here; these were demonstrated to be lower than $2.5 \%$.

\subsubsection{Fe XIII}

Radiative decay rates from Young (2004) for the 27 levels of the $3 s^{2} 3 p^{2}, 3 s 3 p^{3}$, and $3 s^{2} 3 p 3 d$ configurations replace the previous, unpublished calculations of P. R. Young. Comparisons with the earlier calculation are presented in Young (2004), and agreement within $30 \%$ is found for all but two transitions.

\subsection{Phosphorus Isoelectronic Sequence}

$$
\text { 5.14.1. K v }
$$

An ion not previously modeled in CHIANTI, a new electron excitation data set for $\mathrm{K} v$, has recently been published by Wilson $\&$ Bell (2001). The model for the ion includes the five levels of the ground $3 s^{2} 3 p^{3}$ configuration. Effective collision strengths calculated for 10 temperatures over the range $3.3 \leq \log T \leq 5.1$ are from N. J. Wilson (2002, private communication) and were presented in Wilson \& Bell (2001).

Radiative decay rates are from Mendoza \& Zeippen (1982), while experimental energy levels are from the NIST database. 
Applications of the use of the $\mathrm{K} v \lambda 4122.6 / \lambda 4163.3$ ratio to determine the electron density of low-density, nebular plasmas are presented in Keenan et al. (2002).

$$
\text { 5.14.2. Fe XII }
$$

The new data set comprises 143 levels of the lowest $n=3$ configurations. Observed energies (and wavelengths) are from the benchmark study of Del Zanna \& Mason (2005), where many new lines have been identified. Theoretical energies, wavelengths, and $A$-values have been obtained from a SUPERSTRUCTURE calculation that included empirical term energy corrections, described in the same benchmark paper. The $A$-values were calculated with the observed energies whenever available.

The CHIANTI spline fits were obtained from the effective collision strengths calculated in the temperature range $10^{5}-10^{8} \mathrm{~K}$ by Storey et al. (2005). Only transitions from the ground level and those from/to metastable levels have been included.

Storey et al. (2005) performed an $\boldsymbol{R}$-matrix IRON Project calculation. The scattering target included all states belonging to the five energetically lowest electron configurations plus 28 levels of the $3 s^{2} 3 p 3 d^{2}$ configuration. The new collision strengths represent a significant improvement compared to all previous calculations, in particular to the distorted-wave calculations of Flower (1977), which have been widely used in the literature. The main problem with the Flower (1977) calculation was the limited target adopted, which gave rise to inaccurate level mixing and oscillator strengths of some levels by more than an order of magnitude. This in turn affected the cascading to the lower ground levels. Other previous $\boldsymbol{R}$-matrix calculations had various shortcomings, mainly relating to the limited target states. The new calculation appears to have solved long-standing discrepancies such as the high electron densities obtained from Fe XII line ratios and the ratios among the strongest triplets in the EUV (see Storey et al. 2005 and Del Zanna \& Mason 2005 for details).

\subsection{Sulfur Isoelectronic Sequence}

$$
\text { 5.15.1. } \mathrm{Cl} \mathrm{II}
$$

$\mathrm{Cl}_{\text {II }}$ is a new addition to CHIANTI, and the model includes the five levels of the ground $3 s^{2} 3 p^{4}$ configuration. Effective collision strengths for all transitions among these levels were calculated by Wilson \& Bell (2002) using the $\boldsymbol{R}$-matrix method. Data were tabulated for 12 temperatures over the range $3.3 \leq \log T \leq 5.5$, and five-point spline fits were sufficient to fit the data set.

No complete set of $\mathrm{Cl}$ II radiative data is available in the literature, and so a calculation with the code SUPERSTRUCTURE was performed by one of the authors (P. R. Young). The model included the 14 lowest energy configurations: $3 s^{2} 3 p^{4}, 3 s 3 p^{5}$, $3 s^{2} 3 p^{3} 3 d, 3 s^{2} 3 p^{3} 4 l(l=s, p, d, f), 3 s^{2} 3 p^{3} 5 l(l=s, p, d, f, g)$, and $3 s^{2} 3 p^{3} 6 l(l=s, p)$. Observed energy levels from the NIST database were used in the derivation of the $A$-values.

\subsection{Chlorine Isoelectronic Sequence}

\subsubsection{Fe $\mathrm{x}$}

New data replace the previous data for the lowest four configurations in $\mathrm{Fe} x$, corresponding to the 54 fine-structure levels of the lowest and most important $n=3$ configurations. Observed energies (and wavelengths) for these levels are from the benchmark study of Del Zanna et al. (2004), where many new lines have been identified. Theoretical energies, wavelengths, and $A$-values have been obtained from a SUPERSTRUCTURE calculation that included empirical term energy corrections, described in the same benchmark paper. The $A$-values were calculated with the observed energies whenever available.
The CHIANTI spline fits for the 31 lowest levels were obtained from the effective collision strengths calculated in the temperature range $2.5 \times 10^{5}-10^{7} \mathrm{~K}$ by Del Zanna et al. (2004). The authors published the first complete set of effective collision strengths among levels of the $3 s^{2} 3 p^{5}, 3 s 3 p^{6}$, and $3 s^{2} 3 p^{4} 3 d$ configurations. Previous calculations neglected collisional processes involving the metastable levels, which significantly affect the level population even at moderate electron densities. The calculation was carried out in the full Breit-Pauli $\boldsymbol{R}$-matrix approach, including 180 target levels arising from the lowest five configurations. Excitations to the $3 s 3 p^{5} 3 d$ states (levels $31-54$ ) were obtained from the Bhatia \& Doschek (1995) distorted-wave collision strengths, scaled by using the oscillator strengths calculated with a 15-configuration model with SUPERSTRUCTURE.

The rest of the $\mathrm{Fe} x$ atomic model includes configurations $3 s^{2} 3 p^{4} 4 l(l=s, p, d), 3 s^{2} 3 p^{4} 5 l^{\prime}$ and $3 s 3 p^{5} 4 l^{\prime}\left(l^{\prime}=s, p\right)$, and $3 s 3 p^{5} 5 s$, providing 118 additional fine-structure levels, whose atomic data and transition rates are unchanged from version 4 .

\subsection{Argon Isoelectronic Sequence}

\subsubsection{Fe IX}

The atomic data for Fe IX available in earlier versions of CHIANTI have been replaced with the large-scale calculations carried out by Storey et al. (2002). They provide a complete data set of theoretical energy levels, $A$-values, oscillator strengths, and effective collision strengths for the four lowest configurations $3 s^{2} 3 p^{6}, 3 s^{2} 3 p^{5} 3 d, 3 s 3 p^{6} 3 d$, and $3 s^{2} 3 p^{4} 3 d^{2}$, for a total of 140 finestructure levels. Experimental energy levels come from the version 3 of the NIST database (Ralchenko et al. 2005).

The $\boldsymbol{R}$-matrix effective collision strengths provided by Storey et al. (2002) are the most accurate available in the literature and are the first ones to fully include resonances converging on the $3 s^{2} 3 p^{4} 3 d^{2}$ configuration, providing an important contribution to the effective collision strengths of a number of transitions involving the $3 s^{2} 3 p^{5} 3 d$ levels. These previously unaccounted for resonances have large effects on level populations, and alter intensity ratios of lines observed in the EUV range that are used for plasma diagnostics. The large size of the atomic model provided by Storey et al. (2002) also allows us to take into account the effects of radiative cascading, an important contributor to the populations of levels emitting many observed Fe Ix lines.

\subsection{Potassium Isoelectronic Sequence}

$$
\text { 5.18.1. Fe VIII }
$$

Ekberg \& Feldman (2003) measured the energies of a few Fe vIII levels, some of which $\left(3 p^{6} 4 d^{2} D_{J}\right)$ are included in the CHIANTI model for this ion. These energies have been included in the present data set. All the rest of the data are unchanged.

\subsection{Calcium Isoelectronic Sequence}

\subsubsection{Fe VII}

The Fe vII model from version 1 of CHIANTI (Dere et al. 1997) has been completely revised with electron collision and radiative data from Berrington et al. (2000). The model consists of the nine levels of the ground $3 d^{2}$ configuration, and Maxwellianaveraged collision strengths are tabulated for eight temperatures between $\log T=4.3$ and 6.0 (the $\log T_{\max }$ of Fe VII is 5.4; Mazzotta et al. 1998). All transitions between the nine levels have been fitted with five-point splines at all temperatures, to an accuracy of $\leq 1.4 \%$. Observed energy level values are from the NIST database. 
The collision strengths show large differences from the data set previously used in CHIANTI (Keenan \& Norrington 1987), with the new values around 2-3 times larger at $T=T_{\max }$. The differences are due to the larger atomic model used by Berrington et al. (2000) that leads to a larger number of resonances in the collision strengths. There were three errors in the tabulation of $A$-values by Berrington et al. (2000): the values for the $1-4,1-7$, and 4-7 transitions should have been $0.372,0.0150$, and $0.182 \mathrm{~s}^{-1}$.

The $3 d^{2}$ configuration gives rise to transitions at wavelengths $>1480 \AA$ that are found in the spectra of a wide range of astrophysical objects, including planetary nebulae, symbiotic stars, and Seyfert galaxies. Thirteen transitions of Fe VII are present in an archival HST STIS spectrum of the symbiotic star RR Telescopii, and a detailed comparison with the new atomic data listed here is presented in Young et al. (2005a), where good agreement is found.

\section{CHANGES TO THE SOFTWARE}

The suite of IDL routines in version 5 has been modified internally in order to speed the calculation and to include the new processes described in $\S 2$.

The changes introduced to speed the calculations were made necessary by the increasingly larger atomic models now available in CHIANTI, which made the running time of the software of the previous version too long. The routines have been considerably speeded up by introducing array manipulation in place of dealing on a line-to-line basis when calculating the emissivity of each spectral line of each ion. These changes, which have involved several routines, have been introduced without altering the interface with the user, so that nothing has changed in the way CHIANTI routines are used. The benefits in speed are negligible for small atomic models, for which the version 4 software was very fast, but are large for atomic models with more than 100 levels, since the running time has shrunk dramatically from nearly 1 hour per ion to just a few seconds.

The inclusion of ionization and recombination effects in level population has required some more changes. New files have been created (.CILVL and .RECLVL) to store the ionization and recombination rates necessary for this process. A new routine (READ_IONREC.PRO) has been created to read these files and store their data in the input to the routine POP_SOLVER.PRO. This latter routine has been modified to include the correction to the level populations. In case the .CILVL and .RECLVL files are not available, a flag is set in the programs and these processes are ignored. The impact of this new process on the running time is negligible.

However, the introduction of ionization and recombination effects on level population has had a side effect. In previous versions of CHIANTI, the contribution to the intensity of spectral lines from levels below the ionization potential due to cascades from levels above the ionization potential was taken into account in the "dielectronic". WGFA files, which included radiative transitions from the former populated by cascades from the latter.
For the ions for which the complete .RECLVL and .CILVL files are now available (Fe XVII to Fe xxIv), cascades from levels above ionization are now taken into account directly, so that the cascade contribution calculated by the "dielectronic". WGFA files is not anymore necessary. To avoid double-counting this contribution, the transitions from levels below the ionization threshold in the "dielectronic". WGFA files have been given a null wavelength, so they can be removed from the spectrum without having to change the way the "dielectronic" level populations are handled.

\section{SUMMARY}

In the previous sections we have summarized the changes made to CHIANTI that will increase the database completeness and accuracy in the $1-2000 \AA$ range and that will continue to make CHIANTI a vital tool for the interpretation of astrophysical observations.

The main new features of the present version of the database include new physical processes for level population calculation, new extensive calculations for high-energy configurations of highly ionized $\mathrm{Fe}$ ions (Fe IX, Fe XII, Fe xv, Fe XVII-XXIII), new calculations for UV transitions in the C-like, N-like, and O-like sequence, and a complete reassessment of energy levels and line identifications in the 7-18 $\AA$ spectral range. Data for many more ions have also been included.

CHIANTI data and IDL programs are freely available on the Internet at several places in the USA and Europe, ${ }^{1}$ and also through the SolarSoft system. ${ }^{2}$

The work of Enrico Landi is supported by the NNH04AA12I, W10,232, and NNG04ED07P NASA grants. H. E. M. and G. D. Z. acknowledge financial support from PPARC. We warmly thank all the authors who provided us with the data necessary to make this version of the database possible, and all CHIANTI users who helped us improve the database and the software with their comments. We especially wish to thank A. K. Bhatia (NASA/ GSFC) and M. F. Gu (Stanford) for their help in carrying out the calculation of many of the atomic data included in the present version, and for very helpful discussions. The present version of the database would not have been possible without the constant help and discussions of K. J. H. Phillips (NASA/GSFC), who closely collaborated with one of the authors (E. L.) to point out many areas of improvement in the previous version of the database. Useful discussions with J. Sylwester, A. Kepa, and A. Maggio are also gratefully acknowledged. We thank Jan-Uwe Ness and the anonymous referee for their comments, which helped to improve the original manuscript.

\footnotetext{
${ }^{1}$ See http://wwwsolar.nrl.navy.mil/chianti.html, http://www.arcetri.astro.it/ science/chianti/chianti.html, http://www.damtp.cam.ac.uk/user/astro/chianti, http:// www.chianti.rl.ac.uk.

${ }^{2}$ See http://www.lmsal.com/solarsoft.
}

Acton, L. W., Bruner, M. E., Brown, W. A., Fawcett, B. C., Schweizer, W., \& Speer, R. J. 1985, ApJ, 291, 865

Aggarwal, K. M., \& Keenan, F. P. 1994a, J. Phys. B, 27, 2343

1994b, J. Phys. B, 27, 5321

1998, J. Phys. B, 31, 4545

Aggarwal, K. M., \& Kingston, A. E. 1991, Phys. Scr., 44, 517

Alexander, R. D., Clarke, C. J., \& Pringle, J. E. 2005, MNRAS, 358, 283

Argiroffi, C., Drake, J. J., Maggio, A., Peres, G., Sciortino, S., \& Harnden, F. R. 2004, ApJ, 609, 925
Armour, I. A., Bashkin, S., Jelley, N. A., O’Brien, R., Silver, J. D., \& Träbert, E. 1979, J. Phys. (France), 40, C1-211

Armour, I. A., Silver, J. D., \& Träbert, E. 1981, J. Phys. B, 14, 3563

Arthur, S. J. 2001, Rev. Mex. AA Ser. Conf., 10, 49

Badnell, N. R., \& Griffin, D. C. 2001, J. Phys. B, 34, 681

Badnell, N. R., Griffin, D. C., \& Mitnik, D. M. 2001, J. Phys. B, 34, 5071

Baliyan, K. S., \& Bhatia, A. K. 1994, J. Phys. B, 27, 4281

Ballance, C. P., Badnell, N. R., \& Berrington, K. A. 2002, J. Phys. B, 35, 1095

Ballance, C. P., Badnell, N. R., \& Smyth, E. S. 2003, J. Phys. B, 36, 3707 
Bautista, M. A., \& Kallman, T. R. 2001, ApJS, 134, 139

Bautista, M. A., Mendoza, C., Kallman, T. R., \& Palmeri, P. 2003, A\&A, 403, 339

. 2004, A\&A, 418, 1171

Bell, K. L., \& Ramsbottom, C. A. 2000, At. Data Nucl. Data Tables, 76, 176

Bely-Dubau, F., Dubau, J., Faucher, P., \& Gabriel, A. H. 1982a, MNRAS, 198, 239

Bely-Dubau, F., et al. 1982b, MNRAS, 201, 1155

Berrington, K. A., Ballance, C. P., Griffin, D. C., \& Badnell, N. R. 2005,

J. Phys. B, 38, 1667

Berrington, K. A., Nakazaki, S., \& Norrington, P. H. 2000, A\&AS, 142, 313

Berrington, K. A., Saraph, H. E., \& Tully, J. A. 1998, A\&AS, 129, 161

Berrington, K. A., \& Tully, J. A. 1997, A\&AS, 126, 105

Bhatia, A. K., \& Doschek, G. A. 1993, At. Data Nucl. Data Tables, 55, 315 1995, At. Data Nucl. Data Tables, 60, 97

Bhatia, A. K., Feldman, U., \& Doschek, G. A. 1979, A\&A, 80, 22

Bhatia, A. K., \& Landi, E. 2003a, ApJS, 147, 409

$2003 \mathrm{~b}, \mathrm{ApJ}, 585,587$

2003c, At. Data Nucl. Data Tables, 85, 169

Bhatia, A. K., Landi, E., \& Eissner, W. 2006, At. Data Nucl. Data Tables, in press

Bhatia, A. K., Landi, E., \& Mason, H. E. 2003a, At. Data Nucl. Data Tables, 83,71

Bhatia, A. K., Thomas, R. J., \& Landi, E. 2003b, At. Data Nucl. Data Tables, 83,113

Bhatia, A. K., \& Young P. R. 1998, At. Data Nucl. Data Tables, 68, 219

Bloomfield, D. S., Mathioudakis, M., Christian, D. J., Keenan, F. P., \& Linsky, J. L. 2002, A\&A, 390, 219

Bromage, G. E., \& Fawcett, B. C., 1977, MNRAS, 178, 605

Brosius, J. W., Davila, J. M., \& Thomas, R. J. 1998, ApJS, 119, 255

Brown, G. V., Beiersdorfer, P., Liedahl, D. A., Widmann, K., Kahn, S. M., \& Clothiaux, E. J. 2002, ApJS, 140, 589

Brown, G. V., et al. 2005, Phys. Rev. Lett., submitted

Burgess, A., \& Tully J. A. 1992, A\&A, 254, 436

Butler, K., \& Zeippen, C. J. 1994, A\&AS, 108, 1 2001a, A\&A, 372, 1078

2001b, A\&A, 372, 1083

Chidichimo, M. C., Del Zanna, G., Mason, H. E., Badnell, N. R., Tully, J. A., \& Berrington, K. A. 2005, A\&A, 430, 331

Chung, S. M., Drake, J. J., Kashyap, V. L., Lin, L. W., \& Ratzlaff, P. W. 2004, ApJ, 606, 1184

Churilov, S. S., \& Levashov, V. E. 1993, Phys. Scr., 48, 425

Conlon, E. S., Keenan, F. P., \& Aggarwal, K. M. 1992, Phys. Scr., 45, 309

Corrégé, G., \& Hibbert, A. 2002, J. Phys. B, 35, 1211

Crespo Lopez-Urrutia, J. R., Beiersdorfer, P., Savin, D. W., \& Widmann, K. 1998, Phys. Rev. A, 58, 238

Curdt, W., Landi, E., \& Feldman, U. 2004, A\&A, 427, 1045

Danforth, C. W., Blair, W. B., \& Raymond, J. C. 2001, AJ, 122, 938

Daw, A., Parkinson, W. H., Smith, P. L., \& Calamai, A. G. 2000, ApJ, 533, L179

Del Zanna, G. 2006, A\&A, in press

Del Zanna, G., Berrington, K. A., \& Mason, H. E. 2004, A\&A, 422, 731

Del Zanna, G., Chidichimo, M., \& Mason, H. E. 2005, A\&A, 432, 1137

Del Zanna, G., \& Mason, H. E. 2005, A\&A, 433, 731

Dere, K. P., Landi, E., Mason, H. E., Monsignori Fossi, B. C., \& Young, P. R. 1997, A\&AS, 125, 149

Dere, K. P., Landi, E., Young, P. R., \& Del Zanna, G. 2001, ApJS, 134, 331

Dixon, W. V., Sallmen, S., Hurwitz, M., \& Lieu, R. 2001, ApJ, 550, L25

Doron, R., \& Behar, E. 2002, ApJ, 574, 518

Doschek, G. A. 1985, in Autoionization, ed. A. Temkin (New York: Plenum), 171

Doschek, G. A., Feldman, U., \& Cohen, L. 1973, J. Opt. Soc. Am., 63, 1463

Drake, J. J. 2003, ApJ, 594, 496

Drake, J. J., \& Sarna, M. J. 2003, ApJ, 594, L55

Drake, J. J., \& Werner, K. 2005, ApJ, 625, 973

Dubau, J., \& Loulergue, M. 1982, J. Phys. B, 15, 1007

Dupree, A. K., Brickhouse, N. S., Smith, G. H., \& Strader, J. 2005a, ApJ, 625, L131

Dupree, A. K., Lobel, A., Young, P. R., Ake, T. B., Linsky, J. L., \& Redfield, S. 2005b, ApJ, 622, 629

Edelstein, J., Bowyer, S., Korpela, E. J., Lampton, M., Trapero, J., Gomez, J. F., Morales, C., \& Orozco, V. 2001, Ap\&SS, 276, 177

Edlen, B. 1983a, Phys. Scr., 28, 51 1983b, Phys. Scr., 28, 483

1984, Phys. Scr., 30, 135

1985, Phys. Scr., 31, 345

Eissner, W., Galavís, M. E., Mendoza, C., \& Zeippen, C. J. 1999, A\&AS, 137, 165
Eissner, W., Jones, M., \& Nussbaumer, H. 1974, Comput. Phys. Commun., 8, 270

Eissner, W., Landi, E., \& Bhatia, A. K. 2005, At. Data Nucl. Data Tables, 89, 139

Ekberg, J. O., \& Feldman, U. 2003, ApJ, 595, 517

Engström, L., et al. 1980, Phys. Scr., 22, 570

Fawcett, B. C. 1986, At. Data Nucl. Data Tables, 34, 215

Fawcett, B. C., Gabriel, A. H., \& Paget, T. M. 1971, J. Phys. B, 4, 986

Fawcett, B. C., \& Hayes, R. W. 1975, MNRAS, 170, 185

Fawcett, B. C., Phillips, K. J. H., Jordan, C., \& Lemen, J. R. 1987, MNRAS, 225,1013

Feldman, P. D., Ake, T. B., Berman, A. F., Moos, H. W., Sahnow, D. J., Strobel, D. F., Weaver, H. A., \& Young, P. R. 2001, ApJ, 554, L123

Feldman, P. D., Strobel, D. F., Moos, H. W., \& Weaver, H. A. 2004, ApJ, 601, 583

Feldman, U., Curdt, W., Doschek, G. A., Schühle, U., Wilhelm, K., \& Lemaire, P. 1998, ApJ, 503, 467

Feldman, U., Curdt, W., Landi, E., \& Wilhelm, K. 2000, ApJ, 544, 508

Flower, D. R. 1977, A\&A, 54, 163

Froese Fischer, C., \& Saha, H. P. 1983, Phys. Rev. A, 28, 3169

Fuhr, J. R., et al. 1999, NIST Atomic Spectra Database, Version 2.0, NIST Physical Reference Data (Gaithersburg: NIST Physics Laboratory)

Fuhrmeister, B., Schmitt, J. H. M. M., \& Wichmann, R. 2004, A\&A, 417, 701

Gabriel, A. H., \& Paget, T. M. 1972, J. Phys. B, 5, 673

Galavís, M. E., Mendoza, C., \& Zeippen, C. J. 1997, A\&AS, 123, 159

Goett, S. J., \& Sampson, D. H. 1983, At. Data Nucl. Data Tables, 29, 535

Gondoin, P. 2004, A\&A, 426, 1035

Gu, M. F. 2003, ApJ, 582, 1241

Hartigan, P., Edwards, S., \& Pierson, R. 2004, ApJ, 609, 261

Herald, J. E., Bianchi, L., \& Hillier, D. J. 2005, ApJ, 627, 424

Herbert, F., Gladstone, G. R., \& Ballester, G. E. 2001, J. Geophys. Res., 106, 26293

Hibbert, A. 1980, J. Phys. B, 13, 1721

Hibbert, A., Brage, T., \& Fleming, J. 2002, MNRAS, 333, 885

Howley, S. L., et al. 2003, ApJ, 597, 535

Hurwitz, M., Sasseen, T. P., \& Sirk, M. M. 2005, ApJ, 623, 911

Hutton, R., Reistad, N., Engström, L., \& Huldt, S. 1985, Phys. Scr., 31, 506

Jordan, C., Sim, S. A., McMurry, A. D., \& Aruvel, M. 2001, MNRAS, 326, 303

Jupén, C., \& Engström, L. 1997, Phys. Scr., 56, 592

Kashyap, V., \& Drake, J. J. 2002, in ASP Conf. Ser. 277, Stellar Coronae in the Chandra and XMM-Newton Era, ed. F. Favata \& J. J. Drake (San Francisco: ASP), 509

Kastner, S. O., \& Bhatia, A. K. 1979, A\&A, 71, 211

Kato, T., Lang, J., \& Berrington, K. A. 1990, At. Data Nucl. Data Tables, 44, 133

Kato, T., Safronova, U. I., Shlyaptseva, A. S., Cornille, M., Dubau, J., \& Nilsen, J. 1997, At. Data Nucl. Data Tables, 67, 225

Kaufman, V., Sugar, J., \& Cooper, D. 1982, Phys. Scr., 26, 163

Keenan, F. P., Aller, L. H., Espey, B. R., Exter, K. M., Hyung, S., Keenan, M. T. C., Pollacco, D. L., \& Ryans, R. S. I. 2002, Proc. Natl. Acad. Sci., 99, 4152

Keenan, F. P., \& Norrington, P. H. 1987, A\&A, 181, 370

Kelly, R. L. 1987, Atomic and Ionic Spectrum Lines below 2000 Angstroms: Hydrogen through Krypton (J. Phys. Chem. Ref. Data 16, Suppl. 1; New York: AIP)

Kink, I., \& Engström, L. 1997, Phys. Scr., 56, 31 1999, Phys. Scr., 59, 355

Kink, I., Jupén, C., Engström, L., Feldman, U., Laming, J. M., \& Schuehle, U. 1997, ApJ, 487, 956

Kohl, J. L., et al. 1997, Sol. Phys., 175, 613

Kucera, T. A., Feldman, U., Widing, K. G., \& Curdt, W. 2000, ApJ, 538, 424

Lamzin, S. A., Kravtsova, A. S., Romanova, M. M., \& Batalha, C. 2004 Astron. Lett., 30, 413

Lamzin, S. A., Stempels, H. C., \& Piskunov, N. E. 2001, A\&A, 369, 965

Landi, E. 2005, A\&A, 434, 365

Landi, E., \& Bhatia, A. K. 2003a, ApJS, 149, 251 2003b, ApJ, 589, 1075

2005a, At. Data Nucl. Data Tables, 89, 195 2005b, At. Data Nucl. Data Tables, 90, 177 2006a, At. Data Nucl. Data Tables, in press 2006b, At. Data Nucl. Data Tables, in press 2006c, At. Data Nucl. Data Tables, in press

Landi, E., \& Gu, M. F. 2006a, ApJ, in press 2006b, ApJ, in press

Landi, E., \& Landini, M. 2002, A\&A, 384, 1124

Landi, E., Landini, M., Dere, K. P., Young, P. R., \& Mason, H. E. 1999, A\&AS, 135,339 
Landi, E., \& Phillips, K. J. H. 2005, ApJS, 160, 286

Landi, E., Storey, P. J., \& Zeippen, C. J. 2004, ApJ, 607, 640

Lee, T. L., \& Hwang, C. Y. 2004, ApJ, 605, 689

Lepson, J. K., Beiersdorfer, P., Behar, E., \& Kahn, S. M. 2003, ApJ, 590, 604 2005, ApJ, 625, 1045

Lepson, J. K., et al. 2002, ApJ, 578, 648

Lin, C. D., Johnson, W. R., \& Dalgarno, A. 1977, Phys. Rev. A, 15, 154

Maggio, A., Drake, J. J., Kashyap, V., Harnden, F. R., Jr., Micela, G., Peres, G., \& Sciortino, S. 2004, ApJ, 613, 548

Martin, W. C., Sugar, J., Musgrove, A., \& Dalton, G. R. 1995, NIST Atomic Spectra Database, Version 1.0, NIST Physical Reference Data (Gaithersburg: NIST Physics Laboratory)

Matranga, M., Mathioudakis, M., Kay, H. R. M., \& Keenan, F. P. 2005, ApJ, 621, L125

Matsumoto, C., Lieghly, K. M., \& Marshall, H. L. 2004, ApJ, 603, 456

Mauche, C. W., \& Raymond, J. C. 2000, ApJ, 541, 924

May, M. J., et al. 2005, ApJS, 158, 230

Mazzotta, P., Mazzitelli, G., Colafrancesco, S., \& Vittorio, N. 1998, A\&AS, 133,403

McLaughlin, B. M., \& Bell, K. L. 2000, J. Phys. B, 33, 597

McLaughlin, B. M., \& Kirby, K. P. 2001, J. Phys. B, 34, 2255

Mendoza, C., Kallman, T. R., Bautista, M. A., \& Palmeri, P. 2004, A\&A, 414, 377

Mendoza, C., \& Zeippen, C. J. 1982, MNRAS, 198, 127

Merkelis, G., Martinson, I., Kiselius, R., \& Vilkas, M. J. 1999, Phys. Scr., 59, 122

Merkelis, G., Vilkas, M. J., Kiselius, R., \& Gaigalas, G. 1997, Phys. Scr., 56, 41

Mewe, R., Gronenschild, E. H. B. M., \& van den Oord, G. H. J. 1985, A\&AS, 62, 197

Mitnik, D. M., Griffin, D. C., \& Badnell, N. R. 2001, J. Phys. B, 34, 4455

Neill, P. A., Träbert, E., Beiersdorfer, P., Brown, G. V., Harris, C. K., Utter, S. B., \& Wong, L. 2000, Phys. Scr., 62, 141

Ness, J. U., Schmitt, J. H. M. M., Burwitz, V., Mewe, R., \& Predehl, P. 2002, A\&A, 387, 1032

Nussbaumer, H., \& Rusca, C. 1979, A\&A, 72, 129

Pagano, I., Linsky, J. L., Valenti, J., \& Duncan, D. K. 2004, A\&A, 415, 331

Palmeri, P., Mendoza, C., Kallman, T. R., \& Bautista, M. A. 2003a, A\&A, 403, 1175

Palmeri, P., Mendoza, C., Kallman, T. R., Bautista, M. A., \& Melendez, M. 2003b, A\&A, 410, 359

Penston, M. V., et al. 1983, MNRAS, 202, 833

Petz, A., Hauschildt, P. H., Ness, J. U., \& Starrfield, S. 2005, A\&A, 431, 321

Phillips, K. J. H., Dubau, J., Sylwester, J., \& Sylwester, B. 2006, ApJ, in press

Phillips, K. J. H., Mathiudakis, M., Huenemorder, D. P., Williams, D. R., Phillips, M. E., \& Keenan, F. P. 2001, MNRAS, 325, 1500

Phillips, K. J. H., Sylwester, J., Sylwester, B., \& Landi, E. 2003, ApJ, 589, L113

Raga, A. C., Noriega-Crespo, A., \& Velazquez, P. F. 2002, ApJ, 576, L149

Raga, A. C., Velazquez, P. F., Cantó, J., Masciadri, E., \& Rodriguez, L. F. 2001, ApJ, 559, L33

Ralchenko, Y., Jou, F. C., Kelleher, D. E., Kramida, A. E., Musgrove, A., Reader, J., Wiese, W. L., \& Olsen, K. 2005, NIST Atomic Spectra Database, Version 3.0, NIST Physical Reference Data (Gaithersburg: NIST Physics Laboratory)

Ramsbottom, C. A., \& Bell, K. L. 1997, A\&AS, 125, 543

Reader, J., \& Sugar, J. 1975, J. Phys. Chem. Ref. Data, 4, 353

Richter, H., Wood, P. R., Woitke, P., Bolick, U., \& Sedlmayr, E. 2003, A\&A, 400,319

Robinson, R. D., Linsky, J. L., Woodgate, B. E., \& Timothy, J. G. 2001, ApJ, 554,368

Sampson, D. H., Goett, S. J., \& Clark, R. E. H. 1983, At. Data Nucl. Data Tables, 29, 467
Sampson, D. H., Zhang, H. L., \& Fontes, C. J. 1991, At. Data Nucl. Data Tables, 48, 25

Sandlin, G. D., Bruekner, G. E., Scherrer, V. E., \& Tousey, R. 1976, ApJ, 205, L47

Sankrit, R., Blair, W. P., \& Raymond, J. C. 2003, ApJ, 589, 242

Scelsi, L., Maggio, A., Peres, G., \& Gondoin, P. 2004, A\&A, 413, 643

Schmidt, H. T., et al. 1994, Phys. Rev. Lett., 72, 1616

Schmitt, J. H. M. M., \& Ness, J. U. 2004, A\&A, 415, 1099

Shirai, T., Sugar, J., Musgrove, A., \& Wiese, W. L. 2000, Spectral Data for Highly Ionized Atoms: Ti, V, Cr, Mn, Fe, Co, Ni, Cu, Kr, and Mo (J. Phys. Chem. Ref. Data, Monogr. 8; New York: AIP)

Sim, S. A., \& Jordan, C. 2003, MNRAS, 346, 846

Smith, R. K., Brickhouse, N. S., Liedahl, D. A., \& Raymond, J. C. 2001, ApJ, 556, L91

Smith, R. K., \& Cox, D. P. 2001, ApJS, 134, 283

Stefanelli, G. S., Beiersdorfer, P., Decaux, V., \& Widmann, K. 1995, Phys. Rev. A, 52,3651

Storey, P. J., Del Zanna, G., Mason, H. E., \& Zeippen, C. 2005, A\&A, 433, 717

Storey, P. J., \& Zeippen, C. J. 2000, MNRAS, 312, 813

Storey, P. J., Zeippen, C. J., \& Le Dorneuf, M. 2002, A\&A, 394, 753

Swartz, D. A., Ghosh, K. K., Suleimanov, V., Tennant, A. F., \& Wu, K. 2002, ApJ, 574, 382

Sylwester, B., Sylwester, J., Siarkowski, M., Phillips, K. J. H., \& Landi, E. 2004, in IAU Symp. 223, Multi-Wavelength Investigations of Solar Activity, ed. A. V. Stepanov, E. E. Benevolenskaya, \& A. G. Kosovichev (Cambridge: Cambridge Univ. Press), 671

Sylwester, J., et al. 2005, Sol. Phys., 226, 45

Tachiev, G., \& Froese Fischer, C. 1999, J. Phys. B, 32, 5805

2000, J. Phys. B, 33, 2419

2002, A\&A, 385, 716

Tayal, S. S. 1999, J. Phys. B, 32, 5311

Tayal, S. S., \& Hibbert, A. 1984, J. Phys. B, 17, 3835

Thomas, R. J., \& Neupert, W. M. 1994, ApJS, 91, 461

Träbert, E., Beriersdorfer, P., Brown, G. V., Smith, A. J., Utter, S. B., Gu, M. F., \& Savin, D. W. 1999a, Phys. Rev. A, 60, 2034

Träbert, E., Wolf, A., Linkemann, J., \& Tordoir, X. 1999b, J. Phys. B, 32, 537

Velazquez, P. F., Koenigsberger, G., \& Raga, A. C. 2003, ApJ, 584, 284

Velazquez, P. F., Martinell, J. J., Raga, A. C., \& Giacani, E. B. 2004, ApJ, 601, 885

Vernazza, J. E., \& Reeves, E. M. 1978, ApJS, 37, 485

Walter, F. M., et al. 2003, AJ, 126, 3076

Werner, K., Rauch, T., Barstow, M. A., \& Kruk, J. W. 2004a, A\&A, 421, 1169

Werner, K., Rauch, T., Reiff, E., Kruk, J. W., \& Napiwotzki, R. 2004b, A\&A, 427,685

Whiteford, A. D., Badnell, N. R., Ballance, C. P., Loch, S. D., O’Mullane, M. G., \& Summers, H. P. 2002, J. Phys. B, 35, 3729

Wilson, N. J., \& Bell, K. L. 2001, MNRAS, 325, 159

. 2002, MNRAS, 331, 389

Young, P. R. 2004, A\&A, 417, 785

Young, P. R., Berrington, K. A., \& Lobel, A. 2005a, A\&A, 432, 665

Young, P. R., Del Zanna, G., Landi, E., Dere, K. P., Mason, H. E., \& Landini, M. 2003, ApJS, 144, 135

Young, P. R., Dupree, A. K., Espey, B. R., Kenyon, S. J., \& Ake, T. B. 2005b, ApJ, 618, 891

Young, P. R., Landi, E., \& Thomas, R. J. 1998, A\&A, 329, 291

Zhang, H. L., Graziani, M., \& Pradhan, A. K. 1994, A\&A, 283, 319

Zhang, H. L., \& Sampson, D. H. 1987, ApJS, 63, 487

1996, At. Data Nucl. Data Tables, 63, 275

1999, At. Data Nucl. Data Tables, 72, 153

2002, At. Data Nucl. Data Tables, 82, 357

Zou, Y., \& Froese Fischer, C. 2001, J. Phys. B, 34, 915 